\title{
High-SIR Transmission Capacity of Wireless Networks with General Fading and Node Distribution
}

\author{
Radha Krishna Ganti, Jeffrey G. Andrews, and Martin Haenggi
}

\begin{abstract}
In many wireless systems, interference is the main performance-limiting factor, and is primarily dictated by the locations of concurrent transmitters. In many earlier works, the locations of the transmitters is often modeled as a Poisson point process for analytical tractability. While analytically convenient, the PPP only accurately models networks whose nodes are placed independently and use ALOHA as the channel access protocol, which preserves the independence. Correlations between transmitter locations in non-Poisson networks, which model intelligent access protocols, makes the outage analysis extremely difficult. In this paper, we take an alternative approach and focus on an asymptotic regime where the density of interferers $\eta$ goes to 0 . We prove for general node distributions and fading statistics that the success probability $\mathrm{P}_{\mathrm{s}} \sim 1-\gamma \eta^{\kappa}$ for $\eta \rightarrow 0$, and provide values of $\gamma$ and $\kappa$ for a number of important special cases. We show that $\kappa$ is lower bounded by 1 and upper bounded by a value that depends on the path loss exponent and the fading. This new analytical framework is then used to characterize the transmission capacity of a very general class of networks, defined as the maximum spatial density of active links given an outage constraint.
\end{abstract}

\section{INTRODUCTION}

Network information theory attempts to characterize fundamental limits on networked systems of nodes, in particular the rate at which information can propagate through the network between an arbitrary set of nodes. In a wireless network, the first order effect governing the flow of information is the spatial separation between the nodes, which determines the desired signal and interference power at each receiver. Since for large networks, most known information-theoretic approaches lead either to loose upper bounds or are typically lacking in tractability, a considerable number of alternative approaches that lead to characterizations of basic properties of wireless networks are under active investigation.

R. K. Ganti and J. G. Andrews are with the University of Texas at Austin, TX, USA, and M. Haenggi is with the University of Notre Dame, IN, USA. The contact author is R. K. Ganti, rganti@austin.utexas.edu. Date revised: August 15, 2018 
Considerable recent interest has been paid to the impact that the user geometry - i.e., the locations of the transmitters and receivers - has on fundamental metrics of wireless network performance [1], [2]. Since it is impractical (and in most cases, impossible) to enumerate all such locations, it is typical to model their coordinates as drawn from a random two-dimensional point process. The most commonly used spatial random process is the homogeneous Poisson point process (PPP), which is by far the most tractable and well understood, as it presumes independence between each and every node location, as well as a homogeneous distribution over space. Even if the node locations are in fact independent and uniformly scattered in space, most "good" scheduling algorithms induce correlations in the transmitter locations to avoid collisions. This has led to a sharp tradeoff between tractability - requiring essentially an interference-agnostic ALOHA multiple access control (MAC) protocol that preserves the independence - and optimality/generality, whereby more intelligent MAC protocols can be characterized despite introducing dependence and therefore requiring a more general point process.

The objective of this paper is to provide a new mathematical framework for analyzing a very general class of wireless networks. In particular, this paper provides novel tools for modeling interference, computing outage and transmission capacity for a general spatial distribution of transmitters, and under a general fading model.

\section{A. Background and Related Work}

Among the first papers to use a PPP to model the node locations in a wireless network is [3] where the outage probability of $n$ users uniformly distributed in a bounded area $A \subset \mathbb{R}^{2}$ was found with no channel fading, by scaling $A$ and $n$ appropriately. The interference distribution in a Poisson network was shown [4] to be a stable distribution with parameter $2 / \alpha$, where $\alpha$ is the path loss exponent. Both these results as well as subsequent analysis of Poisson networks hinge mainly on two mathematical tools: the probability generating functional (PGFL) and the Palm characterization. Both of these are fairly simple for a PPP because of the independence between the node locations. The conditional PGFL is known only for a PPP (and a few variants), and this has made it extremely difficult to extend the rich set of results on PPPs to other more general spatial node distributions.

In the domain of PPP-modeled networks, significant recent work has developed sophisticated mathematical models on the interference experienced in such networks [5]-[13]. In such cases it is then possible to compute the probability that a typical receiver has a signal-to-noise-interference ratio below some threshold $\theta$, which is termed the outage probability [14], [15]. A final related metric is the transmission capacity (TC), which is the maximum number of successful transmissions per unit area at a specified target outage probability [16], [17]. In many of these early works, interference is typically treated as noise, but this assumption can be relaxed. Transmit/receive approaches that are known to be optimal in 
some multiuser settings, such as dirty-paper coding [18] or successive interference cancellation [19]-[21], can be considered. In this paper, however, interference is treated as noise.

There has been significant interest in moving beyond the Poisson process, but progress has been slow, and is limited to either a deterministic arrangement of nodes (degenerate point processes) or variants of a Poisson point process. In [22], the outage and TC are obtained when the nodes are distributed as a Poisson cluster process, which results from independent clustering applied to a Poisson point process. Carrier sense multiple access (CSMA) MAC protocol results in networks, where the concurrent transmitters have a minimum distance, and this precludes PPP as a model for transmitter locations. Lattice networks are commonly used to model CSMA networks [23], and the outage probability was analyzed in [24][27]. Analyzing CSMA protocol with a random node distribution is extremely difficult and usually the interfering transmitters are approximated by a Poisson point process. In [2], the outage probability was obtained by approximating interferer locations in a CSMA protocol, with a non-homogeneous Poisson point process and in [28]-[30] by excluding interferers in guard zone around the typical transmitter in a Poisson model.

\section{B. Overview of Contributions and Organization}

Since obtaining outage probability (or TC) for a general class of node distributions is extremely difficult, we concentrate on the low-interference regime, which corresponds to a low-density of concurrent transmitters. For any spatial distribution of the transmitters, we show how to obtain two constants $\gamma$ and $\kappa$, so that we can characterize the success probability as

$$
\mathrm{P}_{\mathrm{s}} \sim 1-\gamma \eta^{\kappa}, \quad \eta \rightarrow 0
$$

where $1-\mathrm{P}_{\mathrm{S}}$ is the outage probability of a typical link with the SIR model and $\eta$ is the density of concurrent transmitters ${ }^{1}$. If the two constants $\gamma$ and $\kappa$ can be calculated, then the TC defined in (6) is asymptotically

$$
\mathrm{TC}(\epsilon) \sim\left(\frac{\epsilon}{\gamma}\right)^{1 / \kappa}(1-\epsilon), \quad \epsilon \rightarrow 0
$$

where $\epsilon$ represents the constraint on the outage probability. By the continuity of $\mathrm{P}_{\mathrm{s}}$, the above approximation will be accurate for $\eta$ close to zero.

Unlike the exact characterization of the outage probability, obtaining $\gamma$ and $\kappa$ turns out to be possible for a wide range of MAC protocols and node distributions. In [31]-[33], the outage probability was also approximated in a similar spirit but only for Rayleigh fading, where it was shown that $\kappa=1$ for any ALOHA network and $\kappa=\alpha / 2$ for CSMA, where $\alpha$ is the path loss exponent. This paper however proves

\footnotetext{
${ }^{1}$ By $f(x) \sim g(x), x \rightarrow 0$ we mean that $\lim _{x \rightarrow 0} \frac{f(x)}{g(x)}=1$.
} 
such a result under a general fading model, and provides much more general tools for future non-Poisson network analysis. The transmission capacity is also derived, and compared to prior results, which are nearly exclusively for a PPP. More precisely, this paper makes the following four primary contributions.

1) For any plausible MAC protocol, the interference scaling exponent $\kappa$ is shown to be between 1 and $\alpha \nu / 2$, where $\alpha$ is the path loss exponent, and $\nu \geq 1$ depends only on the fading distribution of a typical link.

2) For any general fading and underlying node distribution with ALOHA, it is shown that $\kappa=1$, and $\gamma$ is shown to depend only on the second-order product density of the spatial distribution of nodes. The TC is then calculated, and is shown to linearly scale with the outage constraint $\epsilon$.

3) The asymptotic outage and the TC for a CSMA network modeled by a Matern hard-core point process with a general fading distribution is obtained, in which $\kappa=\alpha \nu / 2$. From (2), the TC of the CSMA network is observed to be $\Theta\left(\epsilon^{\frac{2}{\alpha \nu}}\right)$ which is a significant improvement compared over ALOHA networks whose TC is $\Theta(\epsilon)$.

4) The asymptotic TC of any spatial distribution of transmitting nodes with Rayleigh fading is obtained and shown to depend only on the second-order product density of the transmit point process.

Finally, we note that although the focus here is on outage and transmission capacity, the techniques are general and can be extended to other SINR-related metrics like expected forward progress [14].

The paper is organized as follows. In Section II, we introduce the system model, the assumptions on the source-destination distribution and the transmitter point process. In Section III, we obtain the bounds on $\kappa$. ALOHA networks are analyzed in Section IV where we show that $\kappa=1$ and provide the exact value of $\gamma$; CSMA networks are studied in Section V. In Section VI, we consider the asymptotic outage results of ALOHA and CSMA networks with thermal noise. In Section VII, we derive the transmission capacity for ALOHA, CSMA networks, and a general class of networks with Rayleigh fading. The notation and symbols used are summarized in Table I.

\section{System Model AND Metrics}

We model the location of the potential transmitters by a stationary and isotropic [34], [35] point process $\Phi \subset \mathbb{R}^{2}$ of unit density on the plane. We assume that the MAC protocol schedules a transmitter set $\Phi_{t} \subset \Phi$ with the following properties:

1) The transmitter set $\Phi_{t}$ is stationary and isotropic.

2) The density of the transmitter set is $\eta \leq 1$, and the MAC protocol can reduce the density $\eta$ to zero.

The large scale path loss model is denoted by $\ell(x): \mathbb{R}^{2} \rightarrow[0, \infty]$ and is assumed to have the following properties:

1) $\ell(x)$ is a non-increasing function of $\|x\|$. 


\begin{tabular}{|c|c|}
\hline Symbol & Description \\
\hline$\Phi$ & point process representing potential transmitters \\
\hline$\Phi_{t} \subset \Phi$ & transmitters selected by the MAC protocol \\
\hline$\eta$ & density of transmitters $\Phi_{t}$ \\
\hline$\rho^{(2)}(x)$ & second-order product density of $\Phi$ \\
\hline$\rho_{\eta}^{(k)}\left(x_{1}, \ldots, x_{k-1}\right)$ & $k$-th order product density of $\Phi_{t}$ \\
\hline $\mathbb{P}^{! o}(A)$ & reduced Palm measure of an event $A$ \\
\hline $\mathbb{E}^{! o}$ & expectation w.r.t. reduced Palm measure \\
\hline$\theta$ & SIR threshold \\
\hline $\mathrm{P}_{\mathrm{s}}$ & success probability, i.e., $\mathbb{P}^{! o}(\operatorname{SIR}(o, r(o))>\theta)$ \\
\hline$\ell(x)$ & large-scale path loss function \\
\hline$\alpha$ & path loss exponent \\
\hline$R$ & source-destination (link) distance \\
\hline $\mathrm{W}_{\mathrm{sd}}$ & (power) fading between source and destination \\
\hline $\mathrm{F}_{\mathrm{sd}}(x)$ & CCDF of $\mathrm{W}_{\mathrm{sd}}$, i.e., $\mathbb{P}\left(\mathrm{W}_{\mathrm{sd}}>x\right)$ \\
\hline$\nu$ & $\begin{array}{l}\text { Exponent of } x \text { of the first term } \\
\text { of the Taylor series of } 1-F_{s d}(x)\end{array}$ \\
\hline $\mathrm{h}$ & (power) fading between an interferer and the receiver \\
\hline I & interference \\
\hline$\|x\|$ & norm of $x$ \\
\hline $\mathcal{L}_{X}(s)$ & Laplace transform of the random variable $X$ \\
\hline$o$ & origin \\
\hline$B(x, y)$ & ball of radius $y$ centered around $x$ \\
\hline
\end{tabular}

TABLE I

NOTATION AND SYMBOLS USED IN THIS PAPER

2) $\int_{B(o, \epsilon)^{c}} \ell(x) \mathrm{d} x<\infty$, for all $\epsilon>0$,

where $B(o, \epsilon)$ denotes the ball of radius $\epsilon$ around the origin. In this paper we concentrate on the following two models, but the analysis can be easily extended to other path loss models:

1) Bounded path loss model: $\ell(x)=\left(1+\|x\|^{\alpha}\right)^{-1}, \alpha>2$.

2) Non-bounded path loss model: $\ell(x)=\|x\|^{-\alpha}, \alpha>2$.

For many proofs, the exact form of the path loss model does not matter as long as $\ell(x)=\Theta\left(\|x\|^{-\alpha}\right)$, $\|x\| \rightarrow \infty$.

\section{A. Success Probability}

We pick a typical transmitter from $\Phi_{t}$ and add a new receiver (a test probe to measure interference and outage) in a random direction at a distance $R$. Since the process $\Phi_{t}$ is stationary, we can move the 
typical transmitter to the origin, and we denote its receiver by $r(o)$. The success probability of a typical link is given by

$$
\mathrm{P}_{\mathrm{s}}=\mathbb{P}^{! o}(\operatorname{SIR}(o, r(o))>\theta),
$$

where

$$
\operatorname{SIR}(o, r(o))=\frac{\mathrm{W}_{\mathbf{s d}} \ell(R)}{\sum_{\mathbf{z} \in \Phi_{t}} \mathrm{~h}_{\mathbf{z} r(o)} \ell(\mathbf{z}-r(o))} .
$$

$\mathbb{P}^{! o}$ represents the reduced Palm probability [34] of $\Phi_{t}$, and is equivalent to conditional probability of real-valued random variables. Essentially, $\mathbb{P}^{! o}$ is the conditional probability measure of the point process given that there is a point at the origin, but disregarding that point in all calculations. For the stationary process $\Phi_{t}$, the Palm probability of an event $A$ can be interpreted as

$$
\mathbb{P}^{! o}(A)=\frac{1}{\eta \pi R^{2}} \mathbb{E} \sum_{x \in \Phi_{t} \cap B(o, R)} \mathbf{1}\left(\left(\Phi_{t}+x\right) \backslash\{x\} \in A\right),
$$

where $\Phi_{t}+x$ denotes the translation of $\Phi_{t}$ by $x$. We require the reduced Palm measure since we are considering the point process distribution from the point of view of the typical point at the origin. The received power (after appropriate signal processing) normalized by the path loss $\ell(R)$ is denoted by $\mathrm{W}_{\mathrm{sd}}$, and $\mathrm{h}_{\mathrm{zr}(o)}$ represents the interference power (normalized by the path loss $\ell(\mathrm{z}-r(o))$ ) of a transmitter $\mathrm{z}$ at the receiver $r(o)$ after signal processing. We assume that the random variables $\mathrm{h}_{\mathrm{z} r(o)}$ are i.i.d., and independent of $W_{s d}$. In this paper, the major emphasis is on SIR rather than SINR, since interference is a major debilitating factor in wireless networks, while noise can be combated by increasing the transmit power to the maximum permissible value. As we shall see in Section VI, it turns out that the results for outage considering noise can easily be obtained using the SIR based outage results.

The success probability can be rewritten as

$$
\begin{aligned}
\mathrm{P}_{\mathrm{s}} & =\mathbb{P}^{! o}\left(\mathrm{~W}_{\mathrm{sd}}>\frac{\theta}{\ell(R)} \sum_{\mathrm{z} \in \Phi_{t}} \mathrm{~h}_{\mathrm{z} r(o)} \ell(\mathbf{z}-r(o))\right) \\
& =\mathbb{E}^{! o} \mathrm{~F}_{\mathrm{sd}}\left(\frac{\theta \mathrm{I}}{\ell(R)}\right)
\end{aligned}
$$

where

$$
\mathrm{I}=\sum_{\mathbf{z} \in \Phi_{t}} \mathrm{~h}_{\mathbf{z} r(o)} \ell(\mathbf{z}-r(o))
$$

denotes the interference and $\mathrm{F}_{\mathbf{s d}}(x)$ the CCDF of $\mathrm{W}_{\mathbf{s d}}$. Evaluating $\mathbb{E}^{! o} \mathrm{~F}_{\mathbf{s d}}\left(\frac{\theta \mathrm{I}}{\ell(R)}\right)$ is extremely hard and may not be possible in most cases. For a general $\mathrm{F}_{\mathrm{sd}}$, evaluating $\mathbb{E}^{! o} \mathrm{~F}_{\mathrm{sd}}\left(\frac{\theta \mathrm{I}}{\ell(R)}\right)$ is not easy even when $\Phi_{t}$ is a Poisson point process. To gain insight about the system performance for general MAC protocols and physical layer technologies, we consider the asymptotic regime $\eta \rightarrow 0$. More precisely, we try to find two constants, the interference scaling exponent $\kappa$ and the spatial contention parameter $\gamma$, such that

$$
\mathrm{P}_{\mathrm{s}} \sim 1-\gamma \eta^{\kappa}, \quad \eta \rightarrow 0
$$


If the two constants $\gamma$ and $\kappa$ are determined exactly, then (5) is a good approximation of the success probability near $\eta=0$. In a wireless network, $\eta \rightarrow 0$ corresponds to a high-SIR regime.

\section{B. Transmission Capacity}

The transmission capacity (TC) metric was introduced in [16] to quantify the maximum spatial density of simultaneous transmissions possible for a given outage constraint, and it is given by

$$
\mathrm{TC}(\epsilon)=(1-\epsilon) \sup \left\{\eta: \mathrm{P}_{\mathbf{s}}>1-\epsilon\right\}
$$

In this paper we analyze the transmission capacity in the high-SIR regime when

1) $\mathrm{F}_{\mathrm{sd}}(x)=\exp (-x)$, corresponding to Rayleigh fading, for any MAC protocol or any point process $\Phi_{t}$.

2) $\mathrm{F}_{\mathrm{sd}}(x)$ is not exponential. Here we restrict the MAC protocol to ALOHA and CSMA.

\section{Received Power Distribution}

The received power $W_{s d}$ depends on the small-scale fading, the diversity order of the channel, the transmit power and the signal processing at the receiver. Its complimentary cumulative distribution function $(\mathrm{CCDF})$ is denoted by $\mathrm{F}_{\mathrm{sd}}(x)$. A few illustrative examples of $\mathrm{W}_{\mathrm{sd}}$ are:

- If suppose all the nodes transmit at a constant power, then $\mathrm{W}_{\mathrm{sd}}=\mathrm{h}_{o r(o)}$ and corresponds to the small-scale fading. If the fading is Rayleigh, $W_{s d}$ is exponentially distributed and when the fading is Nakagami-m, $W_{\text {sd }}$ is gamma distributed.

- The nodes in the system may use multiple antennas for communication, in which case the distribution of $\mathrm{W}_{\mathrm{sd}}$ depends on the specific space-time technique used. If suppose the typical receiver $r(o)$ has $M_{r}$ receive antennas and uses receive beamforming, then $\mathrm{W}_{\mathbf{s d}}$ is $\chi^{2}$ distributed with $2 M_{r}$ degrees of freedom and mean $M_{r}$.

To facilitate the analysis, we assume that the $\mathrm{CCDF} \mathrm{F}_{\mathrm{sd}}(x)$ satisfies the following conditions.

A.1 The CCDF is analytic over the entire real line, and has a Taylor series expansion given by

$$
\mathrm{F}_{\mathrm{sd}}(x)=1-c_{o} x^{\nu}+\sum_{k=1}^{\infty} \frac{c_{k}}{k !} x^{k+\nu}, \quad c_{o} \neq 0,
$$

where $\nu \in \mathbb{N}$. The coefficients $c_{k}$ should satisfy

$$
\sum_{k}\left|c_{k}\right| b^{-k}<\infty
$$

for some $b>1$.

A.2 The CCDF is Lipschitz, i.e., there exists a $C>0$ such that for $x, y>0$

$$
\left|\mathrm{F}_{\mathrm{sd}}(x)-\mathrm{F}_{\mathrm{sd}}(y)\right|<C|x-y|
$$


A.3 $\mathbb{E}\left[\mathrm{W}_{\mathrm{sd}}\right]<\infty$, i.e., $\int_{0}^{\infty} \mathrm{F}_{\mathrm{sd}}(x) \mathrm{d} x<\infty$.

The above conditions are satisfied by many distributions of interest. For example, if the source-destination fading is Nakagami-m distributed, then

$$
\mathrm{F}_{\mathrm{sd}}(x)=\frac{\Gamma(m, m x)}{\Gamma(m)}=1-\frac{1}{\Gamma(m)} \sum_{k=0}^{\infty} \frac{(-1)^{k}}{k !} \frac{x^{m+k}}{m+k} .
$$

In this case $\nu=m$ and $c_{k}=(-1)^{k+1} /(m+k)$, which satisfies Condition A.1, for any $b \geq 1$. For Rayleigh fading, $\mathrm{F}_{\mathrm{sd}}(x)=\exp (-x)$ and $c_{k}=(-1)^{k+1}$. In all the above examples, the derivative of $\mathrm{F}_{\mathrm{sd}}(x)$ is bounded and continuous, i.e., $\sup \frac{\mathrm{dF}_{\mathrm{sd}}(x)}{\mathrm{d} x}$ is bounded and hence $\mathrm{F}_{\mathrm{sd}}(x)$ is Lipschitz with a constant $\left\|\frac{\mathrm{dF}_{\mathrm{sd}}(x)}{\mathrm{d} x}\right\|_{\infty}$.

\section{Power Distribution of an Interferer}

The power (after normalizing by the path loss) from the interferer $\mathrm{z} \in \Phi_{t}$ to the receiver $r(o)$ is denoted by $h_{z r(o)}$. We assume that the random variables $h_{z r(o)}$ are i.i.d. and independent of $W_{\mathrm{sd}}$, and we use $\mathrm{h}$ to denote a generic random variable with the distribution of any $\mathrm{h}_{\mathrm{zr}(o)}$. We also assume

A.4 The moments of $\mathrm{h}$ satisfy $\mathbb{E}\left[\mathrm{h}^{n}\right]=O\left(b^{n} n !\right), n \rightarrow \infty$, for some $b>0$.

For example, in the case of a single-antenna system without power control, $\mathrm{h}$ is distributed as the fading (power) coefficient. In the case of Nakagami-m fading we have $\mathbb{E}\left[\mathrm{h}^{n}\right]=m^{-n} \Gamma(n+m)$, which satisfies Condition A.4.

\section{E. Point Processes}

In this subsection, we briefly introduce the notation and definitions related to point processes that we will be using throughout the paper. A detailed treatment can be found in [34]-[37].

Definition 1 (Second-order product density). For a stationary and isotropic point process $\Phi_{t}$ of density $\eta$, the second-order product density $\rho_{\eta}^{(2)}(x)$ is defined by the following relation:

$$
\mathbb{E}^{! o} \sum_{\mathrm{x} \in \Phi_{t}} \mathbf{1}(\mathrm{x} \in B)=\eta^{-1} \int_{B} \rho_{\eta}^{(2)}(x) \mathrm{d} x,
$$

where $B$ is any Borel subset of $\mathbb{R}^{2}$.

Intuitively the second-order product density $\rho_{\eta}^{(2)}(x)$ denotes the probability of finding a pair of points of $\Phi_{t}$ separated exactly by a distance $\|x\|$. For a homogeneous PPP of density $\eta$, the second order product density is $\rho_{\eta}^{(2)}(x)=\eta^{2}$.

Definition 2 (Second-order reduced moment measure). For a stationary and isotropic point process $\Phi_{t}$ of density $\eta$, the second-order reduced moment measure, denoted by $\mathcal{K}_{\eta}(B), B \subset \mathbb{R}^{2}$, is defined as

$$
\mathcal{K}_{\eta}(B)=\eta^{-1} \mathbb{E}^{! o} \sum_{\mathrm{x} \in \Phi_{t}} \mathbf{1}(\mathrm{x} \in B)
$$


From the definitions of $\mathcal{K}_{\eta}(B)$ and $\rho_{\eta}^{(2)}(x)$, it follows that

$$
\mathcal{K}_{\eta}(B)=\eta^{-2} \int_{B} \rho_{\eta}^{(2)}(x) \mathrm{d} x .
$$

The second-order reduced moment measure is a positive and positive-definite measure [35]. Hence for any compact set $B \subset \mathbb{R}^{2}$, there exists a constant $C_{B}$ such that

$$
\mathcal{K}_{\eta}(B+x)<C_{B}, \quad \forall x \in \mathbb{R}^{2} .
$$

The Rippley's $K$-function [34] is a special case of the second-order reduced moment measure, and is defined as $K_{\eta}(r)=\mathcal{K}_{\eta}(B(o, R))$. We now begin with the analysis of the exponent $\kappa$.

\section{BOUNDS ON THE INTERFERENCE SCALING EXPONENT}

At low transmitter density, it is mainly the interference scaling exponent that determines the outage, and a higher exponent $\kappa$ implies better outage performance. From (4) and (5) it follows that

$$
\frac{\mathbb{E}^{! o}\left[\mathrm{~F}_{\mathrm{sd}}(0)-\mathrm{F}_{\mathrm{sd}}(\mathrm{I})\right]}{\eta}=\eta^{\kappa-1} \gamma+o\left(\eta^{\kappa-1}\right) \text {. }
$$

As $\eta \rightarrow 0$, it is intuitive that the interference I becomes small, and hence we can observe that $\kappa$ is related to the derivative of $\mathrm{F}_{\mathrm{sd}}(x)$ at zero. Since $\left.\frac{\mathrm{d} \mathrm{F}_{\mathrm{sd}}(x)}{\mathrm{d} x}\right|_{x=0}$ is the value of the PDF of $\mathrm{W}_{\mathrm{sd}}$ at zero and finite, it follows that $\kappa \geq 1$. We also have an upper bound on $\kappa$, since $\mathrm{F}_{\mathrm{sd}}(x)$ is monotonically decreasing and its derivatives are bounded by Assumptions A.1 to A.4. It is intuitive that a good MAC protocol should spread out the transmissions, and not cluster them in order to avoid collisions. If the MAC protocol is such that the minimum distance between the transmitters does not increase with decreasing density $\eta$, then the resulting transmitting set will have high interference, and hence poor outage performance. The first theorem makes the notion of spreading ${ }^{2}$ transmissions as $\eta \rightarrow 0$ more precise.

Theorem 1. If the transmitting set $\Phi_{t}$ of density $\eta$ is such that

$$
\text { B.1: } \lim _{\eta \rightarrow 0} \sup _{x \in \mathbb{R}^{2}} \mathcal{K}_{\eta}\left(S_{1}+x\right)<\infty
$$

where $S_{1}=[0,1]^{2}$, then $\kappa \geq 1$.

Proof: See Appendix A.

Let $C_{\eta}=\sup _{x \in \mathbb{R}^{2}} \mathcal{K}_{\eta}\left(x+S_{1}\right)$ where $S_{1}=[0,1]^{2}$. Then from a similar argument as in the proof, it is easy to observe that

$$
\mathbb{E}^{! o}\left[\Phi_{t}(B(o, b))\right]=\lambda \eta \mathcal{K}_{\eta}(B(o, b))<\lambda \eta\left\lceil\pi b^{2}\right\rceil C_{\eta}
$$

\footnotetext{
${ }^{2}$ This is different from the MAC induced clustering in CDMA systems [38], [39], where the MAC protocol is optimized for a fixed density of transmissions.
} 
Condition $A .1$ states that $\lim _{\eta \rightarrow 0} C_{\eta}<\infty$, which implies

$$
\mathbb{E}^{! o}\left[\Phi_{t}\left(B\left(o, \eta^{-a}\right)\right] \rightarrow 0 \quad \text { for } a<1 / 2 .\right.
$$

So $A .1$ implies that the average number of points in a ball of radius $b_{\eta}=\eta^{-a}, a<\frac{1}{2}$, goes to zero as the density tends to zero. This condition is violated when the average nearest-interferer distance remains constant with decreasing density $\eta$. See Figure 1 and [31] for examples that violate Condition B.1. If $B .1$ is violated, $\mathrm{P}_{\mathrm{s}}$ is not equal to 1 when $\eta \rightarrow 0$.
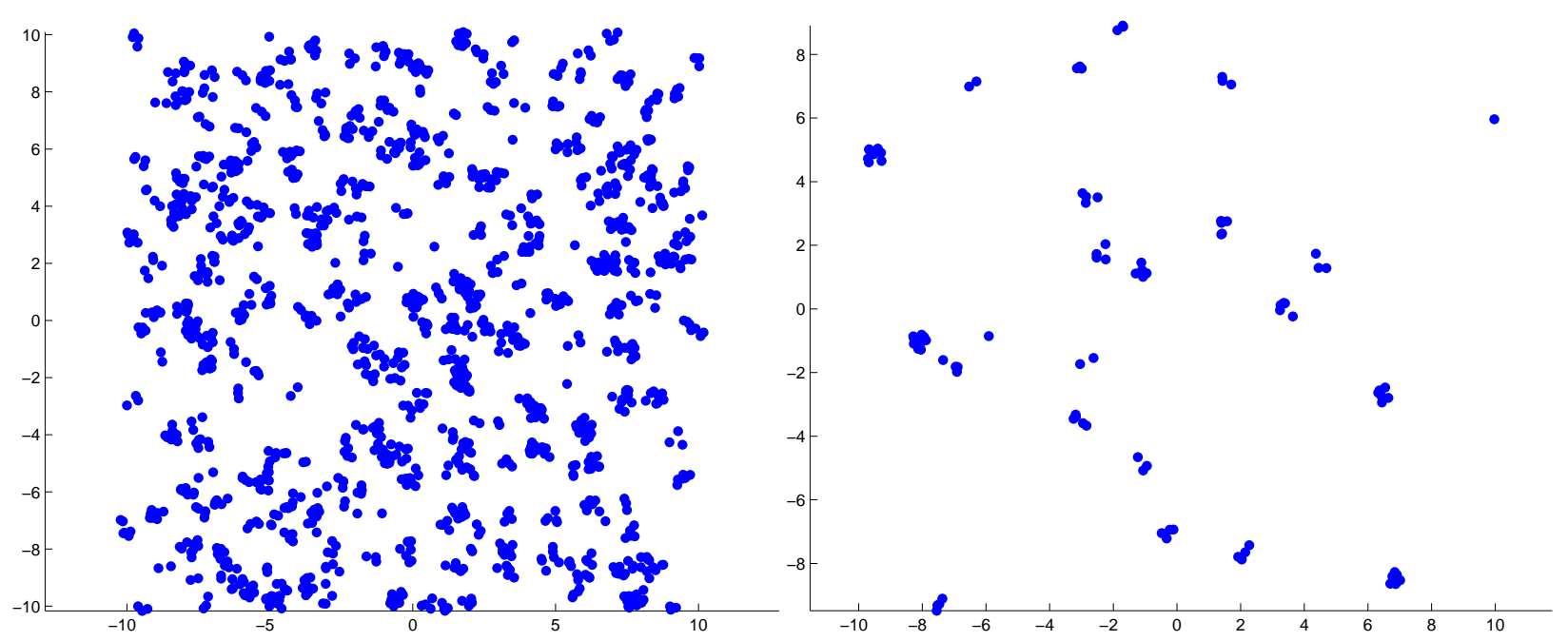

Fig. 1. Illustration of MAC scheme which violates Condition B.1. Left: A network $\Phi$ modeled by a cluster process with a unit density parent process and cluster density 15. Right: The MAC protocol selects a cluster (not individual nodes) to transmit with probability $1 / n$, i.e., $n=15$ resulting in a cluster process $\Phi_{t}$ of density $1 / 15$. We observe that even for $n \rightarrow \infty$, the success probability never approaches one because of intra-cluster interference.

Theorem 2. If the transmit point process $\Phi_{t}$ is such that there exists a $R_{1}>0$ such that

$$
B .2: \quad \lim _{\eta \rightarrow 0} \eta K_{\eta}\left(R_{1} \eta^{-1 / 2}\right)>0,
$$

then $\kappa \leq \frac{\nu \alpha}{2}$.

Proof: See Appendix B.

From Definition 2, $\eta K_{\eta}\left(R_{1} \eta^{-1 / 2}\right)$ is equal to the average number of points in a ball of radius $R_{1} \eta^{-1 / 2}$ and hence Condition $A .2$ requires the number of points inside a ball of radius $R_{1} \eta^{-1 / 2}$ to be greater than zero. Except for pathological cases, this condition is generally valid since the nearest-neighbor distance scales like $\eta^{-1 / 2}$ when the point process has density $\eta$. Essentially any MAC which schedules the nearest interferer only at an average distance $\Theta\left(\eta^{-1 / 2}\right)$ satisfies these two Conditions B.1 and B.2, 
as most common MAC schemes do. Henceforth we consider only MAC schemes which satisfy Conditions B.1 and B.2.

We now investigate the scaling behavior of some common protocols, and the interference exponent $\kappa$ and $\gamma$ values they achieve. The average interference is

$$
\begin{aligned}
\mathbb{E}^{! o}[\mathrm{I}] & \left.=\mathbb{E}^{! o} \sum_{\mathbf{z} \in \Phi_{t}} \mathrm{~h}_{\mathbf{z} r(o)} \ell(\mathbf{z}-r(o))\right) \\
& \stackrel{(a)}{=} \mathbb{E}[\mathrm{h}] \eta^{-1} \int_{\mathbb{R}^{2}} \rho_{\eta}^{(2)}(x) \ell(x-r(o)) \mathrm{d} x,
\end{aligned}
$$

where $(a)$ follows from the definition of the second-order product density. The analysis of the scaling behavior of $\mathrm{P}_{\mathrm{S}}$ depends on whether the average interference is finite or not. The success probability is $\mathbb{E}^{! o} \mathrm{~F}_{\mathrm{Sd}}(\theta \mathrm{I} / \ell(r))$, and if $\mathrm{I}$ is finite almost surely, then the Taylor series expansion of $\mathrm{F}_{\mathrm{sd}}(x)$ can be used to obtain the asymptotics. On the other hand, when $\mathbb{E}^{! o}[I]=\infty$, then the Taylor series expansion cannot be used. For example consider an ALOHA Poisson network with a path loss model $\ell(x)=\|x\|^{-\alpha}$ and Rayleigh fading. As $\rho_{\eta}^{(2)}(x)=\eta^{2}$ for a PPP, the average interference from (11) is

$$
\mathbb{E}^{! o}[\mathrm{I}]=\eta \mathbb{E}[\mathrm{h}] \int_{\mathbb{R}^{2}}\|x\|^{-\alpha} \mathrm{d} x=\infty
$$

Hence, the Taylor series expansion of $\mathrm{F}_{\mathrm{sd}}(x)$ cannot be used. But nevertheless, in this case [14]

$$
\mathrm{P}_{\mathrm{s}}=\exp \left(-\eta R^{2} \theta^{2 / \alpha} C(\alpha)\right) \sim 1-\eta R^{2} \theta^{2 / \alpha} C(\alpha), \quad \eta \rightarrow 0,
$$

where $C(\alpha)=\pi \Gamma(1-2 / \alpha) \Gamma(1+2 / \alpha)$, which shows that $\kappa=1$. In the next two sections, we analyze the outage probability when the transmitting set $\Phi_{t}$ is selected from $\Phi$ using ALOHA and CSMA, respectively. As we shall see, these two MAC protocols achieve the extremes of the interference scaling exponent, with ALOHA resulting in $\kappa=1$ and CSMA in $\kappa=\alpha \nu / 2$.

\section{ALOHA NETWORKS}

The completely decentralized nature of the ALOHA protocol makes it appealing for a wireless network and particularly suitable for an ad hoc network. The independent scheduling of the ALOHA process also makes its analysis much easier than other plausible MAC protocols like CSMA that introduce correlations between the transmit node locations. ALOHA is a natural model when the underlying node distribution is a PPP, since independently thinning a PPP results in a PPP of lower density. Not surprisingly, this combination of PPP with the ALOHA MAC protocol has been extensively analyzed, and closed-form expressions for outage have been obtained for both SISO and MIMO systems [14], [40]. 


\section{A. Asymptotic Success Probability}

An ALOHA network consists of a stationary point process $\Phi$ (need not be a PPP) of unit density, where each node decides to transmit independently of every other node with probability $\eta$. The resulting transmitter set $\Phi_{t}$ is a stationary point process of density $\eta$, and the interference is

$$
\mathrm{I}=\sum_{\mathrm{x} \in \Phi} \mathrm{h}_{\mathrm{x} r(o)} \ell(\mathrm{x}-r(o)) \mathbf{1}\left(\mathrm{x} \in \Phi_{t}\right),
$$

where $1\left(\mathrm{x} \in \Phi_{t}\right)=1$ when the node $\mathrm{x}$ is selected by the ALOHA MAC protocol to transmit.

First, as an example, consider a point process $\Phi=\left\{\mathrm{x}_{1}, \mathrm{x}_{2}\right\}$ composed of only two points (which is a non-stationary network but suitable to illustrate our key idea). Let $Y_{\mathrm{x}}=\mathrm{h}_{\mathrm{x} r(o)} \ell(\mathrm{x}-r(o)), \forall \mathrm{x} \in \Phi$. After thinning $\Phi$ with ALOHA, the interference is

$$
\mathrm{I}=Y_{1} \mathbf{1}\left(\mathrm{x}_{1} \in \Phi_{t}\right)+Y_{2} \mathbf{1}\left(\mathrm{x}_{2} \in \Phi_{t}\right) .
$$

Then, conditioned on $Y_{1}$ and $Y_{2}$, by averaging $\mathrm{F}_{\mathrm{sd}}(\mathrm{I})$ with respect to the ALOHA indicator random variables,

$$
\mathbb{E}_{\mathbf{s d}}(\mathrm{I})=(1-\eta)^{2} \mathrm{~F}_{\mathbf{s d}}(0)+\eta(1-\eta)\left(\mathrm{F}_{\mathbf{s d}}\left(Y_{1}\right)+\mathrm{F}_{\mathbf{s d}}\left(Y_{2}\right)\right)+\eta^{2} \mathrm{~F}_{\mathbf{s d}}\left(Y_{1}+Y_{2}\right)
$$

Since $\mathrm{F}_{\mathrm{sd}}(0)=1$,

$$
\mathbb{E}_{\mathbf{S d}}(\mathrm{I})=1-\eta \sum_{i=1}^{2}\left(1-\mathrm{F}_{\mathbf{s d}}\left(Y_{i}\right)\right)+o(\eta), \quad \eta \rightarrow 0 .
$$

Then averaging with respect to $Y_{i}$ (i.e., fading and point process $\Phi$ ), we obtain that $\kappa=1$ and $\gamma=$ $\mathbb{E}^{! o} \sum_{i=1}^{2}\left(1-\mathrm{F}_{\mathrm{sd}}\left(Y_{i}\right)\right)$. Theorem 3 formalizes the above idea and shows that the interference scaling exponent $\kappa$ is always 1 for ALOHA.

Theorem 3. When ALOHA is used as the MAC protocol, and a node from a stationary point process $\Phi$ is allowed to transmit with probability $\eta$, the success probability is

$$
\mathrm{P}_{\mathrm{s}} \sim 1-\eta \gamma_{\mathrm{ALOHA}}, \quad \eta \rightarrow 0,
$$

where

$$
\gamma_{\mathrm{ALOHA}}=\int_{\mathbb{R}^{2}}\left[1-\mathbb{E}_{\mathrm{h}} \mathrm{F}_{\text {sd }}\left(\mathrm{h} \frac{\theta \ell(x-r(o))}{\ell(R)}\right)\right] \rho^{(2)}(x) \mathrm{d} x,
$$

and $\rho^{(2)}(x)$ is the second-order product density of the initial point process $\Phi$.

Proof: See Appendix C.

The above theorem indicates that $\kappa=1$ irrespective of the point process $\Phi$ and $\mathrm{F}_{\mathrm{sd}}(x)$. The initial point process $\Phi$ affects the asymptotic probability only by its second-order product density. It is also evident that the channel diversity (by using multiple antennas) does not change the exponent $\kappa$, but only affects the pre-constant $\gamma_{\text {ALOHA }}$. 
Intuition: An independently thinned stationary point process converges weakly to a PPP as the density tends to zero [35]. Hence, as $\eta \rightarrow 0$, ALOHA results in a point process that closely resembles a PPP. The result follows since $\kappa=1$ for a PPP. From Theorem 3,

$$
\begin{aligned}
1-\mathrm{P}_{\mathrm{s}} & \sim \eta \int_{\mathbb{R}^{2}}\left[1-\mathbb{E}_{\mathrm{h}} \mathrm{F}_{\mathrm{sd}}\left(\mathrm{h} \frac{\theta \ell(x-r(o))}{\ell(R)}\right)\right] \rho^{(2)}(x) \mathrm{d} x \\
& =\mathbb{E}^{! o} \sum_{\mathrm{x} \in \Phi_{t}} 1-\mathbb{E}_{\mathrm{h}} \mathrm{F}_{\mathrm{sd}}\left(\mathrm{h} \frac{\theta \ell(\mathrm{x}-r(o))}{\ell(R)}\right) .
\end{aligned}
$$

Observe that $1-\mathbb{E}_{\mathrm{h}} \mathrm{F}_{\mathrm{sd}}\left(\mathrm{h} \frac{\theta \ell(x-r(o))}{\ell(R)}\right)$ is the outage caused at the receiver $r(o)$ by a single interferer located at $x$. Hence, in the low-density regime, the overall outage is equal to the sum of the outages caused by each interferer. To evaluate the transmission capacity, the following lemma, which addresses the monotonicity of $\mathrm{P}_{\mathrm{s}}$, is required.

Lemma 1. Let $\mathrm{P}_{\mathrm{s}}(\eta)$ denote the success probability when the ALOHA parameter is $\eta$. Then for any stationary point process $\Phi$,

$$
\eta_{1} \geq \eta_{2} \Rightarrow \mathrm{P}_{\mathbf{s}}\left(\eta_{1}\right) \leq \mathrm{P}_{\mathbf{s}}\left(\eta_{2}\right)
$$

Proof: The proof follows from a standard uniform coupling argument [41] and is omitted.

In an ALOHA network, from the definition of $\gamma_{\mathrm{ALOHA}}$, we observe that if $\rho_{1}^{(2)}(x)<\rho_{2}^{(2)}(x), \forall x$ then $\gamma_{1}$ is smaller than $\gamma_{2}$. So starting with a point process with a guaranteed minimum distance (such as the Matern hard-core process) results in a better outage performance than a Poisson network. For a Poisson cluster process of unit density $\rho^{(2)}(x)>1$, and hence the $\gamma$ for a cluster process is higher than for a PPP, resulting in a worse outage performance.

\section{B. Example: Poisson ALOHA Networks}

We now provide a few examples and evaluate $\gamma_{\mathrm{ALOHA}}$ when the initial point process $\Phi$ is a PPP. When $\Phi$ is a Poisson point process of unit density, the second-order product density is equal to $\rho^{(2)}(x)=1$, and the constant $\gamma_{\mathrm{ALOHA}}$ can be evaluated for different $\mathrm{F}_{\mathrm{sd}}(x)$ using Theorem 3.

1) Nakagami-m fading: When the fading is Nakagami-m distributed, $\mathrm{F}_{\mathrm{sd}}(x)$ is the CCDF of the gamma distribution with m-degrees of freedom, given by

$$
\mathrm{F}_{\mathrm{sd}}(x)=\frac{\Gamma(m, m x)}{\Gamma(m)} .
$$

When $\ell(x)=\|x\|^{-\alpha}$,

$$
\gamma_{\mathrm{ALOHA}}(m)=\frac{\pi \theta^{2 / \alpha} R^{2} \Gamma(m-2 / \alpha) \Gamma(m+2 / \alpha)}{\Gamma(m)^{2}}, \quad m \geq 1 .
$$

This constant has also been calculated in [40]. We observe that $\gamma_{\mathrm{ALOHA}}(m)$ decreases to $\pi$ as $m \rightarrow \infty$, which shows that the outage performance improves as the amount of fading decreases. 
2) Receiver beamforming: When every transmitter uses a single antenna for transmission, each receiver is equipped with $M_{r}$ receive antennas, and receive beamforming is used, the resulting $\mathrm{W}_{\mathbf{s d}}$ is $\chi^{2}$ distributed with $2 M_{r}$ degrees of freedom, and

$$
\mathrm{F}_{\mathrm{sd}}(x)=\frac{\Gamma\left(M_{r}, x\right)}{\Gamma\left(M_{r}\right)}
$$

For $\ell(x)=\|x\|^{-\alpha}$, and Rayleigh fading

$$
\gamma_{\text {ALOHA }}\left(M_{r}\right)=\frac{\pi \theta^{2 / \alpha} R^{2} \Gamma\left(M_{r}-2 / \alpha\right) \Gamma(1+2 / \alpha)}{\Gamma\left(M_{r}\right)}, \quad M_{r} \geq 1 .
$$

Since $\gamma_{\text {ALOHA }}\left(M_{r}\right) \approx \pi \theta^{2 / \alpha} R^{2} \Gamma(1+2 / \alpha) M_{r}^{-2 / \alpha}$, the gain of using multiple receive antennas is $\Theta\left(M_{r}^{-2 / \alpha}\right)$, resulting in a lower outage probability for large $M_{r}$.

\section{CSMA NETWORKS}

We now consider MAC protocols which result in transmitter point processes $\Phi_{t}$ that have a minimum distance between the transmitters. In this case $\mathbb{E}[\mathrm{I}]<\infty$, and we use the Taylor series expansion of $\mathrm{F}_{\mathrm{sd}}(x)$ to obtain the scaling of the success probability. For the sake of illustration, consider only one interferer at a distance $\eta^{-1}$ from $r(o)$ and let $\mathrm{F}_{\mathbf{s d}}(x)=1-x^{2}+x^{3}+o\left(x^{3}\right), x \rightarrow 0$. Neglecting the fading of the interferers, observe that

$$
\mathrm{F}_{\mathrm{sd}}(\mathrm{I})=1-\eta^{\alpha}+\eta^{3 \alpha / 2}=1-\eta^{\alpha}+o\left(\eta^{\alpha}\right)
$$

which shows that $\kappa=\alpha$. In this section, we formalize this procedure to obtain the asymptotic behavior. We use CSMA as a representative MAC protocol and obtain the asymptotic outage probability. For other MAC protocols that impose a minimum distance between transmitters, this proof technique can be easily adapted.

\section{A. CSMA Model}

Although the spatial distribution of the transmitters that concurrently transmit in a CSMA network is difficult to determine, the transmitting set can be closely approximated by a Matern hard-core process [2], [34]. Start with a Poisson point process $\Phi$ of unit density. To each node $\mathrm{x} \in \Phi$, associate a mark $m_{\mathrm{x}}$, a uniform random variable in $[0,1]$. The contention neighborhood of a node $\mathrm{x}$ is the set of nodes which result in an interference power of at least $\mathrm{P}$ at $\mathrm{x}$, i.e.,

$$
\overline{\mathcal{N}}(\mathrm{x})=\{\mathrm{y} \in \Phi: \ell(\mathrm{y}-\mathrm{x})>\mathrm{P}\}
$$

Observe that we are not including fading in the selection of the contention neighborhood. An alternative model has been proposed in [2] which incorporates the fading between the nodes for the selection of the contention set. A node $\mathrm{x} \in \Phi$ belongs to the final CSMA transmitting set if

$$
m_{\mathrm{x}}<m_{\mathrm{y}}, \quad \forall \mathrm{y} \in \overline{\mathcal{N}}(\mathrm{x})
$$


Define $\mathrm{a}=\ell^{-1}(\mathrm{P})$. See Fig. 2 for an illustration of Matern hard-core process. The average number of
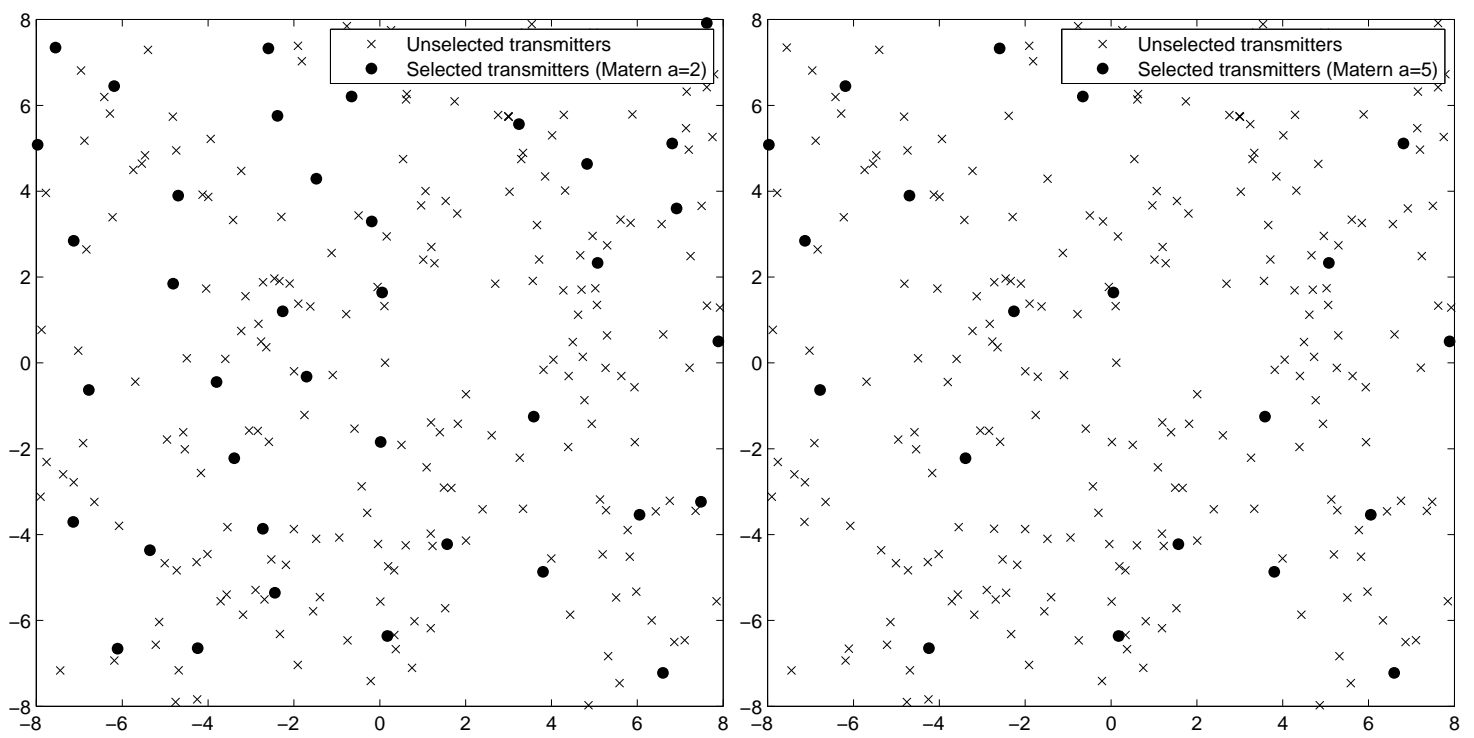

Fig. 2. Left: Matern hard-core process with $\mathrm{a}=2$. Right: Matern hard-core process with $\mathrm{a}=5$. Observe that the minimum distance between nodes increases with a.

nodes in the contention neighborhood of $\mathrm{x} \in \Phi$ does not depend on the location $\mathrm{x}$ by the stationarity of $\Phi$ and is equal to [2]

$$
\mathcal{N}=\mathbb{E}[|\overline{\mathcal{N}}(x)|]=\pi \mathrm{a}^{2}
$$

The density of the CSMA Matern process $\Phi_{t}$ is

$$
\eta=\left[\frac{1-\exp (-\mathcal{N})}{\mathcal{N}}\right]
$$

Let $\rho_{\mathrm{a}}^{(k)}\left(x_{1}, \ldots x_{k-1}\right)$ denote the $k$-th order product density of the Matern hard-core point process $\Phi_{t}$. Let $\mathbf{V}_{\mathrm{a}}\left(x_{1}, \ldots, x_{n}\right)$ denote the volume of the intersection of discs of radius a centered around $x_{i}$ with the convention $\mathbf{V}_{\mathrm{a}}\left(x_{i}\right)=\pi \mathrm{a}^{2}$. Let $x_{k}$ denote the origin $o$, and for any indexing set $J=\left\{a_{1}, \ldots, a_{|J|}\right\}$, and let $\mathbf{V}_{\mathrm{a}}(J)=\mathbf{V}_{\mathrm{a}}\left(x_{a_{1}} \ldots x_{a_{|J|}}\right)$. The following lemma characterizes the product density and its scaling behavior.

Lemma 2. The $k$-th order product density satisfies

$$
\rho_{\mathrm{a}}^{(k)}\left(\mathrm{a} x_{1}, \ldots \mathrm{a} x_{k-1}\right) \sim \mathrm{a}^{-2 k} \tilde{\rho}^{(k)}\left(x_{1}, \ldots x_{k}\right), \quad \mathrm{a} \rightarrow \infty,
$$

where

$$
\begin{gathered}
\tilde{\rho}^{(k)}\left(x_{1}, \ldots, x_{k-1}\right)=k ! \int_{\mathbb{R}^{k}} \exp \left(-\sum_{J \subset\{1, \ldots, k\}}(-1)^{|J|+1} m_{\min J} \mathbf{V}_{1}(J)\right) \\
\cdot \mathbf{1}\left(0 \leq m_{1} \leq \cdots \leq m_{k}<\infty\right) \mathrm{d} m_{1} \ldots \mathrm{d} m_{k} .
\end{gathered}
$$


for $\left\|x_{i}\right\|>1,1 \leq i \leq k-1$, and 0 otherwise.

Proof: See Appendix D

\section{B. Asymptotic Success Probability}

We now present the main result that characterizes the outage behavior of a CSMA system at low densities. Before that, we require the following notation. Let $\mathcal{P}(n)$ denote the set of partitions of $n$, i.e., the set of integers $\left(p_{1}, p_{2} \ldots, p_{k}\right)$, such that $\sum p_{i}=n$ and $1 \leq p_{i} \leq n$. Tuples with different ordering are not distinguished. For example $\mathcal{P}(2)=\{(2),(1,1)\}$, and $\mathcal{P}(4)=\{(4),(1,1,1,1),(1,2,1),(3,1),(2,2)\}$.

Theorem 4. When the set of transmitters forms a Matern CSMA process of density $\eta$ and the Conditions A.1, A.2 and A.4 are satisfied, then

$$
\mathrm{P}_{\mathrm{s}} \sim 1-c_{0} \pi\left(\frac{\theta}{\ell(R)}\right)^{\nu} \eta^{\alpha \nu / 2} A_{I}, \quad \eta \rightarrow 0
$$

where $A_{I}$ is given by

$$
\sum_{\left(p_{1}, \ldots, p_{k}\right) \in \mathcal{P}(\nu)} \int_{\mathbb{R}^{2 k}} \tilde{\rho}^{(k+1)}\left(x_{1}, \ldots, x_{k}\right) \prod_{i=1}^{k}\left\|x_{i}\right\|^{-\alpha p_{i}} \mathbb{E}\left[\mathrm{h}^{p_{i}}\right] \mathrm{d} x_{i},
$$

where $\tilde{\rho}^{(k+1)}\left(x_{1}, \ldots, x_{k}\right), 1 \leq k \leq \nu$, is given in Lemma 2 .

Proof: The proof is presented in Appendix E.

From (17), we observe that the outage probability $1-\mathrm{P}_{\mathrm{s}}$ at low density can be decomposed into three parts:

1) $c_{o}$ stems from the source-destination distribution $\mathrm{CCDF} \mathrm{F}_{\mathrm{sd}}(x)$.

2) The interference-scaling exponent $\kappa=\alpha \nu / 2$ depends on the $\operatorname{CCDF~F}_{\mathbf{s d}}(x)$.

3) $A_{I}$ depends on the point process and the fading between the destination $r(o)$ and the interferers, specifically on the $\nu$ moments of fading $\mathbb{E}\left[\mathrm{h}^{p}\right], 1 \leq p \leq \nu$.

So from a system design perspective, one has to reduce $c_{0}$ and $A_{I}$ for a better outage performance. For a given $\nu$, reducing $A_{I}$ would result from reducing $\mathbb{E}\left[\mathrm{h}^{p}\right], 1 \leq p \leq \nu$. The following Lemma proves the monotonicity of success probability in a CSMA network with respect to the exclusion radius.

Lemma 3. Let $\mathrm{P}_{\mathrm{S}}(\mathrm{a})$ denote the probability of success in a Matern hard-core process when the exclusion radius is a. For a given initial Poisson point process $\Phi$, applying the Matern hard-core process with radii $\mathrm{a}_{1}$ and $\mathrm{a}_{2}$, such that $\mathrm{a}_{1}>\mathrm{a}_{2}$, implies

$$
\mathrm{P}_{\mathrm{s}}\left(\mathrm{a}_{1}\right)>\mathrm{P}_{\mathrm{s}}\left(\mathrm{a}_{2}\right) .
$$


Proof: Fix the marks of $\Phi$ uniformly distributed in $[0,1]$. It is then obvious that the Matern process generated with $a_{1}$ as the exclusion radius is a subset of the Matern process generated with an exclusion radius $\mathrm{a}_{2}$.

The proof of Theorem 4 can be easily adapted to any MAC protocol that results in a transmitter set where the product densities satisfy

$$
\int_{\mathbb{R}^{2 k}} \rho_{\eta}^{(k+1)}\left(x_{1}, \ldots, x_{k}\right) \prod_{i=1}^{k}\left\|x_{i}\right\|^{-\alpha p_{i}} \mathrm{~d} x_{i}<C_{4}^{k} \eta^{\frac{1}{2} \alpha \sum_{i=1}^{k} p_{i}}
$$

for some $C_{4}>0, \forall k \geq 1$, and for all integers $p_{i} \geq 1$. We require bounds on all the product densities in (18) because we are considering arbitrary $\mathrm{F}_{\mathrm{sd}}(x)$. If the function $\mathrm{F}_{\mathrm{sd}}(x)$ has bounds of the type

$$
1-c_{0} x^{\nu} \leq \mathrm{F}_{\mathrm{sd}}(x) \leq 1-c_{0} x^{\nu}+c_{1} x^{\nu+1}
$$

then the asymptotic theorem can be proved by just considering $\nu+2$ product densities. In this case it suffices to prove that $\mathbb{E}^{! o}\left[\mathrm{I}^{\nu+1}\right]=o\left(\mathbb{E}^{! o}\left[\mathrm{I}^{\nu}\right]\right)$ as $\eta \rightarrow 0$. For example, when $\mathrm{F}_{\text {sd }}(x)=\exp (-x)$ we have

$$
1-x \leq \mathrm{F}_{\mathrm{sd}}(x) \leq 1-x+x^{2}, \quad x>0,
$$

and hence the second and the third order product densities are sufficient to evaluate the asymptotics.

\section{EFFECT OF NOISE}

In the previous sections, we have neglected noise in the asymptotic outage analysis. We now discuss how noise changes the value of $\gamma$ and $\kappa$. With thermal noise, the success probability is

$$
\mathrm{P}_{\mathrm{s}}=\mathbb{P}^{! o}\left(\frac{\mathrm{W}_{\mathrm{sd}} \ell(R)}{\mathrm{I}+\frac{\sigma^{2}}{P}}>\theta\right),
$$

where $\sigma^{2}$ is the noise power, and $P$ is the common transmit power. Using the CCDF of $\mathrm{W}_{\mathrm{sd}}$ we obtain

$$
\mathrm{P}_{\mathrm{s}}=\mathbb{E}^{! o} \mathrm{~F}_{\mathrm{sd}}\left(\frac{\theta \mathrm{I}}{\ell(R)}+\mathrm{N}\right)
$$

where $\mathrm{N}=\frac{\sigma^{2} \theta}{P \ell(R)}$. So when the density of transmitters $\eta \rightarrow 0$, we no longer have $\mathrm{P}_{0}$ equal to 1 , where

$$
\mathrm{P}_{0} \triangleq \lim _{\eta \rightarrow 0} \mathrm{P}_{\mathrm{s}}=\mathrm{F}_{\mathrm{sd}}(\mathrm{N})
$$

Hence for small $\eta$, the success probability can be represented as

$$
\mathrm{P}_{\mathrm{s}} \sim \mathrm{P}_{0}-\gamma \eta^{\kappa}, \quad \eta \rightarrow 0
$$

Consider the Taylor series expansion of $\mathrm{F}_{\mathrm{Sd}}(x)$ around $\mathrm{N}$

$$
\mathrm{F}_{\mathrm{sd}}(x+\mathrm{N})=1-c_{o}(\mathrm{~N}) x^{\nu}+\sum_{k=1}^{\infty} \frac{c_{k}(\mathrm{~N})}{k !} x^{k+\nu}, \quad c_{o}(\mathrm{~N}) \neq 0 .
$$

Since $1-\mathrm{F}_{\mathrm{sd}}(y)$ is the CDF, the derivative of $1-\mathrm{F}_{\mathrm{sd}}(y)$ corresponds to the PDF. For any unimodal distribution, the PDF is non-zero at $y \neq 0$, and hence it follows that $\nu=1$ for $\mathrm{N} \neq 0$. The following 
observations can be made concerning the spatial contention parameter $\gamma$ and the interference scaling exponent $\kappa$ when noise is present.

1) Since $\nu=1$, the interference scaling exponent $1 \leq \kappa \leq \alpha / 2$. So unlike the noiseless case, the upper bound on $\kappa$ depends only on the path loss exponent.

2) With ALOHA, the interference scaling exponent $\kappa=1$, while the spatial contention parameter is

$$
\gamma=\int_{\mathbb{R}^{2}}\left[\mathrm{~F}_{\mathrm{sd}}(\mathrm{N})-\mathbb{E}_{\mathrm{h}} \mathrm{F}_{\text {sd }}\left(\mathrm{h} \frac{\theta \ell(x-r(o))}{\ell(R)}+\mathrm{N}\right)\right] \rho^{(2)}(x) \mathrm{d} x .
$$

This can be obtained using techniques similar to the proof of Theorem 3 .

3) When CSMA is used as the MAC protocol, $\kappa=\alpha / 2$, and the spatial contention parameter is

$$
\gamma=\frac{\theta c_{0}(\mathrm{~N}) \pi \mathbb{E}[\mathrm{h}]}{\ell(R)} \int_{\mathbb{R}^{2}} \tilde{\rho}^{(2)}(x)\|x\|^{-\alpha} \mathrm{d} x,
$$

where $\tilde{\rho}^{(2)}(x)$ is given by (15). This result can be obtained from the proof of Theorem 4, by setting $\nu=1$.

Analyzing outage with noise is particularly simple when $\mathrm{F}_{\mathrm{sd}}(x)=\exp (-x)$, i.e., Rayleigh fading. The success probability is

$$
\mathrm{P}_{\mathrm{s}}=\exp \left(-\frac{\theta \mathrm{I}}{\ell(R)}\right) \exp (-\mathrm{N})
$$

Observe that the success probability factorizes into two terms, one that depends only on interference and the other only on noise [14]. Using this factorization, and Theorem 3 it follows that

$$
\gamma=\exp (-\mathrm{N}) \int_{\mathbb{R}^{2}}\left[1-\mathbb{E}_{\mathrm{h}} \mathrm{F}_{\mathrm{sd}}\left(\mathrm{h} \frac{\theta \ell(x-r(o))}{\ell(R)}\right)\right] \rho^{(2)}(x) \mathrm{d} x
$$

which agrees with (21). Similarly it can be verified that $c_{0}(\mathrm{~N})=\exp (-\mathrm{N})$ in (22).

\section{TRANSMISSION CAPACITY}

The transmission capacity (TC) metric captures the global effect of interference on the network throughput. It depends on the spatial distribution of transmitters, includes the MAC protocol, the physical layer technologies used, the path loss function, and the target SIR and outage probability. In short, the TC captures many of the most important features of a wireless network. Since for many MAC protocols, spatial distribution of nodes, and fading distributions, the outage probability itself is not known, finding the exact TC is not feasible. Therefore, we analyze the TC in the asymptotic regime when $\epsilon \rightarrow 0$. More precisely, we try to find an easily computable function $\mathrm{TC}_{1}(\epsilon)$ such that

$$
\mathrm{TC}(\epsilon)=\mathrm{TC}_{1}(\epsilon)+o\left(\mathrm{TC}_{1}(\epsilon)\right), \quad \epsilon \rightarrow 0 .
$$

In the previous sections, we have obtained an asymptotically tight approximation of the success probability of the form

$$
\mathrm{P}_{\mathrm{s}}=1-\gamma \eta^{\kappa}, \quad \eta \rightarrow 0
$$


It is intuitive to expect that the TC can be obtained by inverting the asymptotic of $\mathrm{P}_{\mathrm{s}}$, to solve for the density $\eta$ corresponding to $\mathrm{P}_{\mathrm{s}}=1-\epsilon$. The following lemma formalizes this asymptotic inversion and will be used to characterize the TC.

Lemma 4. Let $f(x)$ and $f_{1}(x)$ be continuous, positive, strictly increasing functions with $f(0)=f_{1}(0)=$ 0 . Furthermore let $f_{1}(x)$ be locally convex at 0 , and

$$
f(x)=f_{1}(x)+o\left(f_{1}(x)\right), \quad x \rightarrow 0 .
$$

Define $f^{*}(\epsilon)=\sup \{x: f(x)<\epsilon\}$, and $f_{1}^{*}(\epsilon)$ similarly. Then

$$
f^{*}(\epsilon)=f_{1}^{*}(\epsilon)+o\left(f_{1}^{*}(\epsilon)\right), \quad \epsilon \rightarrow 0 .
$$

Proof: See Appendix F.

We will now use this Lemma to obtain the asymptotic TC in ALOHA and CSMA networks.

\section{A. TC of ALOHA Networks}

For an ALOHA network $\kappa=1$, and hence the approximation $\gamma \eta$ is linear. The monotonicity of $\mathrm{P}_{\mathrm{s}}$ for ALOHA follows from Lemma 1. Hence all the necessary conditions of Lemma 4 are satisfied in the case of ALOHA networks. Defining $f(\eta)=1-\mathrm{P}_{\mathrm{s}}$, and using the asymptotic approximation of $\mathrm{P}_{\mathrm{s}}$, we can obtain the TC for ALOHA networks from Lemma 4.

Theorem 5. When ALOHA is used as the MAC protocol on a node set distributed as a stationary point process $\Phi$ with second-order product density $\rho^{(2)}(x)$,

$$
\mathrm{TC}(\epsilon)=\frac{\epsilon}{\int_{\mathbb{R}^{2}}\left[1-\mathbb{E F}_{\mathrm{sd}}\left(\mathrm{h} \frac{\theta \ell(x-r(o))}{\ell(R)}\right)\right] \rho^{(2)}(x) \mathrm{d} x}+o(\epsilon)
$$

Proof: Follows from Lemmas 1 and 4 and Theorem 3.

Using Theorem 5, the asymptotic TC of PPP ALOHA networks, when $\mathrm{W}_{\mathrm{sd}}$ and $\mathrm{h}$ are Nakagami-m distributed or $\chi^{2}$ distributed, can be obtained in closed form.

Corollary 3 (Nakagami-m fading). When $\mathrm{W}_{\mathrm{sd}}$ and $\mathrm{h}$ are Nakagami-m distributed, from Theorem 5 the transmission capacity is

$$
\mathrm{TC}(\epsilon)=\frac{\epsilon}{\gamma_{\mathrm{ALOHA}}(m)}+o(\epsilon)
$$

where $\gamma_{\mathrm{ALOHA}}(m)$ is given in (13). 
Corollary 4 (SIMO system with beamforming). When receiver beamforming with $M_{r}$ antennas is used, the TC is

$$
\mathrm{TC}(\epsilon)=\frac{\epsilon}{\gamma_{\mathrm{ALOHA}}\left(M_{r}\right)}+o(\epsilon),
$$

where $\gamma_{\mathrm{ALOHA}}\left(M_{r}\right)$ is given in (14).

This asymptotic TC obtained in both these cases can be verified by comparing them with the results in [40], wherein the TC was obtained using different techniques.

\section{B. TC of CSMA Networks}

Similar to ALOHA networks, the following theorem characterizes the asymptotic TC of CSMA networks.

Theorem 6. When the transmitting set of nodes results from the CSMA MAC protocol described in Section $V$, the TC is

$$
\operatorname{TC}(\epsilon)=\frac{\epsilon^{\frac{2}{\alpha \nu}}}{\left(\pi c_{0} A_{I}\left(\frac{\theta}{\ell(R)}\right)^{\nu}\right)^{\frac{2}{\alpha \nu}}}+o\left(\epsilon^{\frac{2}{\alpha \nu}}\right),
$$

where $A_{I}$ is defined in Theorem 4.

Proof: Follows from Lemmas 3, 4, and Theorem 4.

We note that the TC achieved by the CSMA protocol is $\Theta\left(\epsilon^{\frac{2}{\alpha \nu}}\right)$ compared to $\Theta(\epsilon)$ for ALOHA. We also observe that the TC of CSMA changes by an exponent for MIMO compared to SISO, while such gain is not registered by ALOHA. We have the following simplification in the case of Rayleigh fading.

Corollary 5. For CSMA with Rayleigh fading, i.e., $\mathrm{F}_{\mathbf{s d}}(x)=\exp (-x)$,

$$
\operatorname{TC}(\epsilon)=\left(\frac{\epsilon}{\gamma_{\mathrm{CSMA}}}\right)^{2 / \alpha}+o(\epsilon)
$$

where

$$
\gamma_{\mathrm{CSMA}}=\frac{R^{\alpha} \theta \pi^{\alpha / 2} 2^{3-\alpha}}{\alpha-2}+4 \theta R^{\alpha} \pi^{2} \int_{1 / \sqrt{\pi}}^{2 / \sqrt{\pi}} \frac{r^{1-\alpha}}{g(r)} \mathrm{d} r
$$

and

$$
g(r)=2 \pi-2 \arccos \left(\frac{\sqrt{\pi}}{2} r\right)+\frac{r \sqrt{\pi}}{2} \sqrt{4-\pi r^{2}} .
$$

\section{TC of General Networks with Rayleigh Fading}

In this subsection, we consider a network with a general distribution of nodes, but restrict the CCDF $\mathrm{F}_{\text {sd }}(x)$ to be exponential, and obtain the TC. The distribution of fading between the interferers and the tagged receiver $\mathrm{h}$ can be arbitrary. The results in this subsection are extensions of Theorem 5 and Theorem 6 to general node distributions with exponential distribution for $\mathrm{W}_{\mathrm{sd}}$. 
Define

$$
\begin{aligned}
\Delta(x) & \triangleq 1-\mathcal{L}_{\mathrm{h}}\left(\frac{\theta}{\ell(R)} \ell(x-r(o))\right), \\
\mu_{\eta} & \triangleq \eta^{-1} \int_{\mathbb{R}^{2}} \rho_{\eta}^{(2)}(x) \Delta(x) \mathrm{d} x, \\
\sigma_{\eta} & \triangleq \eta^{-1} \int_{\mathbb{R}^{2} \times \mathbb{R}^{2}} \rho_{\eta}^{(3)}(x, y) \Delta(x) \Delta(y) \mathrm{d} x \mathrm{~d} y,
\end{aligned}
$$

where $\mathcal{L}_{\mathrm{h}}(x)=\mathbb{E}\left[e^{-\mathrm{h} x}\right]$ is the Laplace transform of the random variable $\mathrm{h}$. Since $\mathrm{W}_{\mathrm{sd}}$ is exponential,

$$
\Delta(x)=1-\mathbb{P}\left(\mathrm{W}_{\mathrm{sd}}>\frac{\mathrm{h} \theta \ell(x-r(o))}{\ell(R)}\right) .
$$

Hence, $\Delta(x)$ can be interpreted as the outage caused by a single interferer located at $x$. Obviously $0 \leq \Delta(x) \leq 1$, and when $\mathrm{h}$ is gamma distributed with unit mean (Nakagami-m fading),

$$
\Delta(x)=1-\left(1+\frac{\theta \ell(x-r(o))}{m \ell(R)}\right)^{-m} .
$$

The expectation of $\sum_{\mathrm{x} \in \Phi_{\eta}} \Delta(\mathrm{x})$ is given by $\mu_{\eta}$, and $\sigma_{\eta}$ denotes the average of $\sum_{\mathrm{x}, \mathrm{y} \in \Phi_{\eta}, \mathrm{x} \neq \mathrm{y}} \Delta(\mathrm{x}) \Delta(\mathrm{y})$. We now provide bounds on the success probability $\mathrm{P}_{\mathrm{s}}$ which we later show to be asymptotically tight.

Lemma 5. When $\mathrm{F}_{\mathrm{sd}}(x)=\exp (-x)$, the probability of success is bounded for all $0 \leq \eta \leq 1$,

$$
1-\mu_{\eta} \leq \mathrm{P}_{\mathrm{s}} \leq \min \left\{1-\mu_{\eta}+\frac{\sigma_{\eta}}{2}, \mathcal{G}[\exp (-\Delta(x))]\right\},
$$

where $\mathcal{G}$ is the probability generating functional of $\Phi_{t}$ with respect to its reduced Palm measure.

Proof: The success probability is

$$
\begin{gathered}
\mathrm{P}_{\mathrm{s}}=\mathbb{P}^{! o}\left(\mathrm{~W}_{\mathrm{sd}} \geq \theta \ell(R)^{-1} \sum_{\mathrm{x} \in \Phi_{t}} \mathrm{~h}_{\mathrm{x} r(o)} \ell(\mathrm{x}-r(o))\right) \\
\stackrel{(a)}{=} \mathbb{E}^{! o} \exp \left(-\theta \ell(R)^{-1} \sum_{\mathrm{x} \in \Phi_{t}} \mathrm{~h}_{\mathrm{x} r(o)} \ell(\mathrm{x}-r(o))\right),
\end{gathered}
$$

where $(a)$ follows since $\mathrm{W}_{\mathrm{sd}}$ is an exponential random variable. Taking the expectation with respect to $\mathrm{h}_{\mathrm{x} r(o)}$ in the interference, we obtain

$$
\mathrm{P}_{\mathrm{s}}=\mathbb{E}^{! o}\left[\prod_{\mathrm{x} \in \Phi} 1-\Delta(\mathrm{x})\right] .
$$

We can observe that $0 \leq \Delta(x) \leq 1$, and hence using the inequality

$$
1-\sum a_{i} \leq \prod 1-a_{i} \leq 1-\sum a_{i}+\frac{1}{2} \sum_{i \neq j} a_{i} a_{j},
$$

and the definition of the second- and third-order product densities, we obtain the lower bound $1-\mu$, and the upper bound $1-\mu+\kappa / 2$. The other upper bound can be obtained using the inequality $1-\Delta(\mathrm{x}) \leq$ $\exp (-\Delta(\mathrm{x}))$ and the definition of the probability generating functional. 
Define

$$
\mathrm{TC}_{\mathrm{u}}(\epsilon) \triangleq(1-\epsilon) \sup \left\{\eta: \min \left\{1-\mu_{\eta}+\sigma_{\eta} / 2, \mathcal{G}[\exp (-\Delta(x))]\right\} \geq 1-\epsilon\right\}
$$

and

$$
\mathrm{TC}_{1}(\epsilon) \triangleq(1-\epsilon) \sup \left\{\eta: \mu_{\eta} \leq \epsilon\right\}
$$

It can be easily verified that

$$
\mathrm{TC}_{1}(\epsilon) \leq \mathrm{TC}(\epsilon) \leq \mathrm{TC}_{\mathrm{u}}(\epsilon)
$$

We now present the main theorem of this subsection which characterizes the TC of general networks when $\mathrm{W}_{\mathbf{s d}} \sim \exp (1)$.

Theorem 7. If the transmitter set $\Phi_{t}$ satisfies Condition B.1,

C.1 $\lim _{\eta \rightarrow 0} \frac{\eta^{-1} \int_{\mathbb{R}^{2}} \rho_{\eta}^{(2)}(x) \Delta^{2}(x) \mathrm{d} x}{\sigma_{\eta}}>2$,

C.2 $\sigma_{\eta}=o\left(\mu_{\eta}\right)$,

as $\eta \rightarrow 0$, the transmission capacity when $\mathrm{F}_{\mathrm{sd}}(x)=\exp (-x)$ is

$$
\mathrm{TC}(\epsilon)=\mathrm{TC}_{1}(\epsilon)+o\left(\mathrm{TC}_{1}(\epsilon)\right), \quad \epsilon \rightarrow 0
$$

Proof: See Appendix G.

Remarks:

1) Computing $\mathrm{TC}_{1}(\epsilon)$ is much simpler than computing the actual $\mathrm{TC}$, since $\rho^{(2)}(x)$ is known for many point processes.

2) In the case of ALOHA networks, $\rho_{\eta}^{(2)}(x)=\eta^{2} \rho^{(2)}(x)$, and hence

$$
\mathrm{TC}_{l}(\epsilon)=\frac{\epsilon(1-\epsilon)}{\int_{\mathbb{R}^{2}} \rho^{(2)}(x) \Delta(x) \mathrm{d} x},
$$

which matches Theorem 5 with $\mathrm{F}_{\mathrm{sd}}(x)=\exp (-x)$.

3) Conditions C.1 and C.2 are generally satisfied by most point processes. For example for a PPP of density $\eta$, the second-order product density $\rho_{\eta}^{(2)}(z)=\eta^{2}$, and hence

$$
\mu_{\eta}=\eta \int_{\mathbb{R}^{2}} \Delta(x) \mathrm{d} x
$$

Also, $\rho^{(3)}(x, y)=\eta^{3}$, and so

$$
\sigma_{\eta}=\eta^{2} \int_{\mathbb{R}^{2} \times \mathbb{R}^{2}} \Delta(x) \Delta(y) \mathrm{d} x \mathrm{~d} y=\mu_{\eta}^{2} .
$$

Therefore it follows that $\sigma_{\eta}=o\left(\mu_{\eta}\right)$. Also

$$
\lim _{\eta \rightarrow 0} \frac{\eta^{-1} \int_{\mathbb{R}^{2}} \rho_{\eta}^{(2)}(x) \Delta^{2}(x) \mathrm{d} x}{\sigma_{\eta}}=\lim _{\eta \rightarrow 0} \eta^{-1} \frac{\int_{\mathbb{R}^{2}} \Delta^{2}(x) \mathrm{d} x}{\left(\int_{\mathbb{R}^{2}} \Delta(x)\right)^{2}}=\infty>2,
$$

verifying C.2. In fact, constructing reasonable MAC schemes [32] that lead to a transmitter process $\Phi_{t}$ for which Conditions C.1 and C.2 are violated is difficult-if not impossible. 


\section{CONCLUSIONS}

A new mathematical framework to analyze outage in networks with complex MAC protocols, general spatial node distributions, and general fading is introduced. We define two constants, the interference scaling exponent $\kappa$ and the spatial contention $\gamma$ such that the success probability $\mathrm{P}_{\mathrm{s}} \sim 1-\gamma \eta^{\kappa}, \eta \rightarrow 0$, where $\eta$ is the density of transmitters. By the continuity of $\mathrm{P}_{\mathrm{s}}$, this formulation characterizes the outage probability for small $\eta$. We also use this framework to analyze the transmission capacity. We first show that the interference scaling exponent satisfies $1 \leq \kappa \leq \alpha \nu / 2$, where $\alpha$ is the path loss exponent and $\nu$ depends on the distribution of the received power. For ALOHA we show that $\kappa=1$, irrespective of the spatial node distribution we start with or the channel diversity. We also provide the constant $\gamma$ in this case. The other extreme of the interference scaling exponent, $\kappa=\alpha \nu / 2$, is obtained by the CSMA MAC protocol, for which we also provide the exact value of $\gamma$. The constant $\gamma$ depends only on the product densities of the transmitter point process. Using this framework we also obtain the transmission capacity of ALOHA and CSMA networks for any channel gain distribution. We show that for a CSMA network the TC scales like $\Theta\left(\epsilon \frac{2}{\alpha \nu}\right)$, in contrast to an ALOHA network with a TC of $\Theta(\epsilon)$. When the channel gains are Rayleigh distributed, we obtain the asymptotic TC for any MAC protocol and spatial distribution of nodes. The mathematical techniques introduced in this paper can be used to analyze other relevant metrics for a low density of interferers.

\section{APPENDIX A}

\section{Proof of Theorem 1}

It suffices to prove that $1-\mathrm{P}_{\mathrm{S}}=o\left(\eta^{1-\epsilon}\right)$ for any $\epsilon>0$. For notational convenience, without loss of generality we assume $\theta / \ell(R)=1$. The probability of success is equal to $\mathbb{E}^{! o} \mathrm{~F}_{\text {sd }}(\mathrm{I})$ and hence

$$
\frac{1-\mathrm{P}_{\mathrm{s}}}{\eta}=\frac{\mathbb{E}^{! o}\left[1-\mathrm{F}_{\mathrm{sd}}(\mathrm{I})\right]}{\eta}
$$

Since $\mathrm{F}_{\mathrm{sd}}(x)$ is a Lipschitz function and also a CCDF, we have

$$
\mathrm{F}_{\mathrm{sd}}(x)-\mathrm{F}_{\mathrm{sd}}(x+y) \leq \min \{1, C y\}, \quad y \geq 0 .
$$

Hence we obtain

$$
\frac{\mathbb{E}^{! o}\left[1-\mathrm{F}_{\mathrm{sd}}(\mathrm{I})\right]}{\eta} \leq \frac{\mathbb{E}^{! o}[\min \{1, C \mathrm{I}\}]}{\eta} \leq \frac{\max \{1, C\} \mathbb{E}^{! o}[\min \{1, \mathrm{I}\}]}{\eta}
$$


Define $b=\ell^{-1}(1)$. Define $\Phi_{t}^{\text {near }}=\left\{\mathrm{x} \in \Phi_{t}: \ell(\mathrm{x}-r(o))>1\right\}$, and $\Phi_{t}^{\mathrm{far}}=\left\{\mathrm{x} \in \Phi_{t}: \ell(\mathrm{x}-r(o))<1\right\}$. Then it follows that

$$
\begin{aligned}
\min \{1, \mathrm{I}\} & \leq \min \left\{1, \sum_{x \in \Phi_{t}^{\text {near }}} \mathrm{h}_{\mathrm{x} r(o)} \ell(\mathrm{x}-r(o))+\sum_{\mathrm{x} \in \Phi_{t}^{\mathrm{far}}} \mathrm{h}_{\mathrm{x} r(o)} \ell(\mathrm{x}-r(o))\right\}, \\
& \stackrel{(a)}{\leq} \sum_{x \in \Phi_{t}^{\text {near }}} \min \left\{1, \mathrm{~h}_{\mathrm{x} r(o)} \ell(\mathrm{x}-r(o))\right\}+\sum_{\mathrm{x} \in \Phi_{t}^{\text {far }}} \mathrm{h}_{\mathrm{x} r(o)} \ell(\mathrm{x}-r(o)), \\
& \leq \underbrace{\Phi_{t}(B(r(o), b))}_{\mathrm{I}_{1}}+\underbrace{\sum_{\mathbf{x} \in \Phi_{t}^{\text {far }}} \mathrm{h}_{\mathrm{x} r(o)} \ell(\mathrm{x}-r(o))}_{\mathrm{I}_{2}} .
\end{aligned}
$$

To obtain $(a)$, we have used the fact that $\min \{1, x+y\} \leq \min \{1, x\}+\min \{1, y\}$. Also recall that $\Phi_{t}(B(r(o), b))$ denotes the number of of $\Phi_{t}$ in the set $B(r(o), b)$. Using the definition of the secondorder product density, we obtain

$$
\begin{aligned}
\eta^{-1} \mathbb{E}^{! o}\left[\mathrm{I}_{2}\right] & =\eta^{-1} \mathbb{E}^{! o} \sum_{\mathrm{x} \in \Phi_{t}^{\text {far }}} \mathrm{h}_{\mathrm{x} r(o)} \ell(\mathrm{x}-r(o)) \\
& =\eta^{-1} \mathbb{E}[\mathrm{h}] \mathbb{E}^{! o} \sum_{\mathrm{x} \in \Phi_{t}^{\text {far }}} \ell(\mathrm{x}-r(o)) \\
& \stackrel{(a)}{=} \mathbb{E}[\mathrm{h}] \eta^{-2} \int_{\ell(x-r(o))<1} \rho^{(2)}(x) \ell(x-r(o)) \mathrm{d} x,
\end{aligned}
$$

where $(a)$ follows from the definition of $\rho_{\eta}^{(2)}(x)$. Define $\tilde{\ell}(x)=\ell(x)$, when $\ell(x-r(o))<1$ and $\tilde{\ell}(x)=1$ otherwise. We then have

$$
\begin{aligned}
& \eta^{-2} \int_{\ell(x-r(o))<C} \rho^{(2)}(x) \ell(x-r(o)) \mathrm{d} x \\
& \leq \eta^{-2} \sum_{(k, j) \in \mathbb{Z}^{2}} \int_{S_{k j}} \rho^{(2)}(x) \tilde{\ell}(x-r(o)) \mathrm{d} x,
\end{aligned}
$$

where $S_{k j}=[k, k+1] \times[j, j+1]$. Letting $s_{k j}=\sup \left\{\tilde{\ell}(z-r(o)), z \in S_{k j}\right\}$, we obtain

$$
\begin{aligned}
& \eta^{-2} \int_{\ell(x-r(o))<1} \rho^{(2)}(x) \ell(x-r(o)) \mathrm{d} x \\
& \leq \eta^{-2} \sum_{(k, j) \in \mathbb{Z}^{2}} s_{k j} \int_{S_{k j}} \rho^{(2)}(x) \mathrm{d} x \\
& =\sum_{(k, j) \in \mathbb{Z}^{2}} s_{k j} \mathcal{K}_{\eta}\left(S_{k j}\right) \\
& \stackrel{(a)}{<} C_{S_{1}} \sum_{(k, j) \in \mathbb{Z}^{2}} s_{k j} \stackrel{(b)}{<} \infty
\end{aligned}
$$


where $(a)$ follows from Assumption B.1 and $(b)$ follows since $s_{k j}$ decays like $\left(k^{2}+j^{2}\right)^{-\alpha / 2}$. Now considering the other term in (31),

$$
\begin{aligned}
\eta^{-1} \mathbb{E}^{! o}\left[\mathrm{I}_{1}\right] & =\eta^{-1} \mathbb{E}^{! o} \Phi_{t}(B(r(o), b)) \\
& \stackrel{(a)}{=} \mathcal{K}_{\eta}(B(r(o), b)) \\
& \stackrel{(b)}{<} C_{B(r(o), b)}<\infty,
\end{aligned}
$$

where $(a)$ follows from Definition 2, and (b) follows from (9) and Assumption B.1. Hence it follows from (31) that

$$
\lim _{\eta \rightarrow 0} \frac{1-\mathrm{P}_{\mathrm{s}}}{\eta}<\frac{\mathbb{E}^{! o}[\min \{1, C \mathrm{I}\}]}{\eta}<\infty
$$

proving the theorem.

\section{APPENDIX B}

PRoOF OF THEOREM 2

It suffices to prove that $1-\mathrm{P}_{\mathrm{s}}=\Omega\left(\eta^{\nu \alpha / 2}\right)$. The success probability is

$$
\begin{aligned}
\mathrm{P}_{\mathrm{s}} & =\mathbb{P}^{! o}\left(\mathrm{~W}_{\mathrm{sd}}>\frac{\theta}{\ell(R)} \sum_{\mathbf{z} \in \Phi_{t}} \mathrm{~h}_{\mathbf{z} r(o)} \ell(\mathbf{z}-r(o))\right) \\
& \stackrel{(a)}{\leq} \mathbb{P}^{! o}\left(\mathrm{~W}_{\mathbf{s d}}>\frac{\theta}{\ell(R)} \sum_{\mathbf{z} \in \Phi_{t} \cap B\left(o, R_{1} \eta^{-1 / 2}\right)} \mathrm{h}_{\mathbf{z} r(o)} \ell(\mathbf{z}-r(o))\right) \\
& \stackrel{(b)}{\leq} \mathbb{P}^{! o}\left(\mathrm{~W}_{\mathbf{s d}}>\frac{\theta}{\ell(R)} \ell\left(2 R_{1} \eta^{-1 / 2}\right) X\right),
\end{aligned}
$$

where $X=\sum_{\mathbf{z} \in \Phi_{t} \cap B\left(o, R_{1} \eta^{-1 / 2}\right)} \mathrm{h}_{\mathbf{z r}(o)}$. (a) follows since we have truncated the interference, and $(b)$ follows from the fact that $B\left(r(o), R_{1} \eta^{-1 / 2}\right) \subset B\left(o, 2 R_{1} \eta^{-1 / 2}\right)$ for small $\eta$. Since $\mathrm{h}_{\mathrm{z} r(o)}$ are independent random variables, $X$ can be alternatively written as $X=\sum_{i=1}^{n} \mathrm{~h}_{i}$, where $\mathrm{h}_{i}$ are i.i.d and distributed as $\mathrm{h}_{z r(o)}$, where $n$ is the number of points of $\Phi_{t}$ in the ball $B\left(o, R_{1} \eta^{-1 / 2}\right)$. Hence

$$
\mathrm{P}_{\mathrm{s}} \leq \mathbb{E}^{! o} \mathrm{~F}_{\mathrm{sd}}\left(\frac{\theta}{\ell(R)} \ell\left(2 R_{1} \eta^{-1 / 2}\right) X\right)
$$

Since $\mathrm{F}_{\mathrm{sd}}(x)<1$, by the dominated convergence theorem [42] and the expansion of $\mathrm{F}_{\mathrm{sd}}(x)$ for small $x$,

$$
\lim _{\eta \rightarrow 0} \frac{1-\mathrm{P}_{\mathrm{s}}}{\eta^{\nu \alpha / 2}} \geq \lim _{\eta \rightarrow 0} \eta^{-\nu \alpha / 2} \mathbb{E}^{! o} \Theta\left(\left(\ell\left(2 R_{1} \eta^{-1 / 2}\right) X\right)^{\nu}\right) .
$$

Using the fact that $y^{\nu}, y>0$ is a convex function for $\nu \geq 1$, we have

$$
\lim _{\eta \rightarrow 0} \frac{1-\mathrm{P}_{\mathrm{s}}}{\eta^{\nu \alpha / 2}} \geq \lim _{\eta \rightarrow 0} \eta^{-\nu \alpha / 2} \Theta\left(\left(\ell\left(2 R_{1} \eta^{-1 / 2}\right) \mathbb{E}^{! o}[X]\right)^{\nu}\right) .
$$

In the limit $\ell\left(2 R_{1} \eta^{-1 / 2}\right)^{\nu} \eta^{-\nu \alpha / 2}$ tends to a positive constant and hence it suffices to prove that $\mathbb{E}^{! o}[X]^{\nu}>$ 0 . From the definition of $X$ we have $\mathbb{E}^{! o}[X]=\mathbb{E}^{! o}[n] \mathbb{E}[\mathrm{h}]$, and hence from Assumption B.2, we obtain

$$
\lim _{\eta \rightarrow 0} \mathbb{E}^{! o}[n]=\lim _{\eta \rightarrow 0} \eta K\left(R_{1} \eta^{-1 / 2}\right)>0
$$

hence verifying our claim. 


\section{APPENDIX C}

\section{Proof of THEOREM 3}

Proof: Let $\mathrm{I}_{R} \triangleq \sum_{\mathrm{x} \in \Phi \cap B(o, R)} \mathrm{h}_{\mathrm{x} r(o)} \ell(\mathrm{x}-r(o)) \mathbf{1}\left(\mathrm{x} \in \Phi_{t}\right)$. By the continuity of $\mathrm{F}_{\mathrm{sd}}(x)$ and the dominated convergence theorem, we have

$$
\mathbb{E}^{! o}\left[\mathrm{~F}_{\mathrm{sd}}(\mathrm{I})\right]=\lim _{R \rightarrow \infty} \mathbb{E}^{! o}\left[\mathrm{~F}_{\mathrm{sd}}\left(\mathrm{I}_{R}\right)\right]
$$

Hence we have

$$
\lim _{\eta \rightarrow 0} \frac{1-\mathrm{P}_{\mathrm{s}}}{\eta}=\lim _{\eta \rightarrow 0} \lim _{R \rightarrow \infty} \frac{1-\mathbb{E}^{! o} \mathrm{~F}_{\mathrm{sd}}\left(\mathrm{I}_{R}\right)}{\eta} .
$$

We now show that the limits in the above equation can be exchanged, i.e.,

$$
\lim _{\eta \rightarrow 0} \lim _{R \rightarrow \infty} \frac{1-\mathbb{E}^{! o} \mathrm{~F}_{\text {sd }}\left(\mathrm{I}_{R}\right)}{\eta}=\lim _{R \rightarrow \infty} \lim _{\eta \rightarrow 0} \frac{1-\mathbb{E}^{! o} \mathrm{~F}_{\text {sd }}\left(\mathrm{I}_{R}\right)}{\eta} .
$$

For the limits to be exchanged, we require $1-\mathbb{E}^{! o} \mathrm{~F}_{\mathrm{sd}}\left(I_{R}\right)$ should be uniformly convergent as $R \rightarrow \infty$. Define $\bar{I}_{R}$ by the relation $\mathrm{I}=\mathrm{I}_{R}+\overline{\mathrm{I}}_{R}$. By the Lipschitz property of $\mathrm{F}_{\mathrm{sd}}(x)$ we obtain

$$
\left|\mathbb{E}^{! o}\left[\frac{\mathrm{F}_{\mathrm{sd}}\left(\mathrm{I}_{R}\right)-\mathrm{F}_{\mathrm{sd}}(I)}{\eta}\right]\right|<C \frac{\mathbb{E}^{! o}\left[\bar{I}_{R}\right]}{\eta} .
$$

By the definition of the second order product density, we have

$$
\mathbb{E}^{! o}\left[\bar{I}_{R}\right]=\eta \int_{B(o, R)^{c}} \rho^{(2)}(x) \ell(x-r(o)) \mathrm{d} x,
$$

and hence

$$
\left|\mathbb{E}^{! o}\left[\frac{\mathrm{F}_{\mathrm{sd}}\left(\mathrm{I}_{R}\right)-\mathrm{F}_{\mathrm{sd}}(I)}{\eta}\right]\right|<C \int_{B(o, R)^{c}} \rho^{(2)}(x) \ell(x-r(o)) \mathrm{d} x,
$$

and we observe that the RHS tends to zero at a rate that does not depend on $\eta$. Hence $\left(1-\mathbb{E}^{! o} \mathrm{~F}_{\mathrm{sd}}\left(\mathrm{I}_{R}\right)\right) / \eta$ tends uniformly to $\left(1-\mathbb{E}^{! o} \mathrm{~F}_{\mathrm{sd}}(\mathrm{I})\right) / \eta$, and the limits can be interchanged. Since we are considering only simple point processes the cardinality $|\Phi \cap B(o, R)|<\infty$ almost surely. Define $M: \equiv|\Phi \cap B(o, R)|$. Hence for a finite $R$,

$$
\begin{aligned}
\mathbb{E}^{! o} \mathrm{~F}_{\mathrm{sd}}\left(\mathrm{I}_{R}\right) & =\mathbb{E}^{! o} \sum_{k=0}^{M} \frac{\eta^{k}(1-\eta)^{M-k}}{k !} \\
& \cdot \sum_{1 \leq i_{1}, \ldots, i_{k} \leq M}^{\neq} \mathrm{F}_{\mathrm{sd}}\left(Y_{1}+\cdots+Y_{k}\right),
\end{aligned}
$$

and thus

$$
\lim _{\eta \rightarrow o} \frac{1-\mathbb{E}^{! o} \mathrm{~F}_{\mathrm{sd}}\left(\mathrm{I}_{R}\right)}{\eta}=\mathbb{E}^{! o} \sum_{i=1}^{M}\left(1-\mathrm{F}_{\mathrm{sd}}\left(Y_{i}\right)\right) .
$$

So by the interchangeability of limits,

$$
\lim _{\eta \rightarrow 0} \frac{1-\mathrm{P}_{\mathrm{s}}}{\eta}=\mathbb{E}^{! o} \sum_{\mathrm{x} \in \Phi}\left[1-\mathrm{F}_{\mathrm{sd}}(\mathrm{h} \ell(\mathrm{x}-r(o))],\right.
$$


which by the definition of the second-order product density is

$$
\lim _{\eta \rightarrow 0} \frac{1-\mathrm{P}_{\mathbf{s}}}{\eta}=\int_{\mathbb{R}^{2}}\left[1-\mathbb{E F}_{\mathbf{s d}}(\mathrm{h} \ell(x-r(o)))\right] \rho^{(2)}(x) \mathrm{d} x .
$$

\section{APPENDIX D}

\section{PROOF OF LEMMA 2}

Proof: Let $\mathbf{V}_{\mathrm{a}}\left(x_{1}, \ldots, x_{n}\right)$ denote the volume of the intersection of discs of radius a centered around $x_{i}$ with the convention $\mathbf{V}_{\mathrm{a}}\left(x_{i}\right)=\pi \mathrm{a}^{2}$. Let $x_{k}$ denote the origin $o$. Let

$$
f\left(m_{1}, \ldots, m_{k}\right)=\exp \left(-\sum_{J \subset\{1, \ldots, k\}}(-1)^{|J|+1} m_{\min J} \mathbf{V}_{\mathbf{a}}(J)\right),
$$

where $\mathbf{V}_{\mathrm{a}}(J)=\mathbf{V}_{\mathrm{a}}\left(x_{a_{1}} \ldots x_{a_{|J|}}\right), J=\left\{a_{1}, \ldots, a_{|J|}\right\}$. The $k$-th order product density is [2], [34]

$$
\rho_{\mathrm{a}}^{(k)}\left(x_{1}, \ldots, x_{k-1}\right)=k ! \int f\left(m_{1}, \ldots, m_{k}\right) \mathbf{1}\left(0 \leq m_{1} \leq \cdots \leq m_{k} \leq 1\right) \mathrm{d} m_{1} . . \mathrm{d} m_{k} .
$$

We also have

$$
\mathbf{V}_{\mathrm{a}}\left(\mathrm{a} x_{1}, \ldots, \mathrm{a} x_{m}\right)=\mathrm{a}^{2} \mathbf{V}_{1}\left(x_{1}, \ldots, x_{m}\right)
$$

and hence

$$
\begin{gathered}
\rho_{\mathrm{a}}^{(k)}\left(\mathrm{a} x_{1}, \ldots, \mathrm{a} x_{k-1}\right)=k ! \int \exp \left(-\mathrm{a}^{2} \sum_{J \subset\{1, \ldots, k\}}(-1)^{|J|+1} m_{\min J} \mathbf{V}_{1}(J)\right) \\
\cdot \mathbf{1}\left(0 \leq m_{1} \leq \cdots \leq m_{k} \leq 1\right) \mathrm{d} m_{1} \ldots \mathrm{d} m_{k} .
\end{gathered}
$$

Using the substitution $\mathrm{a}^{2} m_{i} \rightarrow m_{i}$ we obtain

$$
\begin{gathered}
\rho_{\mathrm{a}}^{(k)}\left(\mathrm{a} x_{1}, \ldots, \mathrm{a} x_{k-1}\right)=\mathrm{a}^{-2 k} k ! \int \exp \left(-\sum_{J \subset\{1, \ldots, k\}}(-1)^{|J|+1} m_{\min J} \mathbf{V}_{1}(J)\right) \\
\cdot \mathbf{1}\left(0 \leq m_{1} \leq \cdots \leq m_{k} \leq \mathrm{a}^{2}\right) \mathrm{d} m_{1} \ldots \mathrm{d} m_{k} .
\end{gathered}
$$

Using the dominated convergence theorem, the lemma is proved with

$$
\begin{gathered}
\tilde{\rho}^{(k)}\left(x_{1}, \ldots, x_{k-1}\right)=k ! \int \exp \left(-\sum_{J \subset\{1, \ldots, k\}}(-1)^{|J|+1} m_{\min J} \mathbf{V}_{1}(J)\right) \\
\cdot \mathbf{1}\left(0 \leq m_{1} \leq \cdots \leq m_{k}<\infty\right) \mathrm{d} m_{1} \ldots \mathrm{d} m_{k} .
\end{gathered}
$$




\section{APPENDIX E}

PROOF OF THEOREM 4

Proof: From (7), we observe that

$$
\begin{aligned}
\mathrm{P}_{\mathrm{s}} & =\mathbb{E}^{! o} \mathrm{~F}_{\mathrm{sd}}\left(\frac{\theta}{\ell(R)} \mathrm{I}\right) \\
& =1-c_{0} \mathbb{E}^{! o}\left(\frac{\theta}{\ell(R)} \mathrm{I}\right)^{\nu}+\mathbb{E}^{! o}\left[\sum_{k=1}^{\infty} \frac{c_{k}}{k !}\left(\frac{\theta}{\ell(R)} \mathrm{I}\right)^{k+\nu}\right],
\end{aligned}
$$

So it suffices to prove that

$$
\mathbb{E}^{! o}\left[\sum_{k=1}^{\infty} \frac{c_{k}}{k !}\left(\frac{\theta}{\ell(R)} \mathrm{I}\right)^{k+\nu}\right]=o\left(\mathbb{E}^{! o}(\mathrm{I})^{\nu}\right)
$$

as $\eta \rightarrow 0$. Without loss of generality and for notational convenience we assume $\theta / \ell(R)=1$. We have

$$
\lim _{\mathrm{a} \rightarrow \infty} \frac{\mathrm{P}_{\mathrm{s}}-\left(1-\mathbb{E}^{! o}\left[\mathrm{I}^{\nu}\right]\right)}{\mathrm{a}^{\alpha \nu}}=\lim _{\mathrm{a} \rightarrow \infty} \sum_{k=1}^{\infty} \frac{c_{k}}{k !} \frac{\mathbb{E}^{! o}\left[\mathrm{I}^{k+\nu}\right]}{\mathrm{a}^{\alpha \nu}}
$$

We now prove that there exists some $D_{n}$ such that $\frac{\mathbb{E}^{! o}\left[I^{k+\nu}\right]}{\mathrm{a}^{\alpha \nu / 2}}<D_{k}$, where $D_{k}$ does not depend on a and

$$
\sum\left|D_{k} c_{k}\right| / k !<\infty
$$

Then using the dominated convergence theorem, we can exchange the limit operation and the summation. Let $\mathcal{P}(n)$ denote the set of partitions of $n$, i.e., the set of integer sets $\left(p_{1}, p_{2} \ldots, p_{k}\right)$, such that $\sum p_{i}=n$ and $1<p_{i} \leq n$. Tuples with just different ordering are not distinguished.Then it can be shown that

$$
\begin{aligned}
\eta \mathbb{E}^{! o}\left[\mathrm{I}^{n}\right]= & \sum_{\left(p_{1}, \ldots, p_{k}\right) \in \mathcal{P}(n)} \int \rho_{a}^{(k+1)}\left(x_{1}, \ldots, x_{k}\right) \\
& \cdot \prod_{i=1}^{k} \ell\left(x_{i}\right)^{p_{i}} \mathbb{E}\left[\mathrm{h}^{p_{i}}\right] \mathrm{d} x_{i} .
\end{aligned}
$$

Since $\rho^{(k+1)}\left(x_{1}, \ldots, x_{k}\right)<\prod \mathbf{1}\left(\left\|x_{i}\right\|>\right.$ a $)$ we obtain,

$$
\eta \mathbb{E}^{! o}\left[\mathrm{I}^{n}\right]=\sum_{\left(p_{1}, \ldots, p_{k}\right) \in \mathcal{P}(n)} \int \prod_{i=1}^{k} \ell\left(x_{i}\right)^{p_{i}} \mathbb{E}\left[\mathrm{h}^{p_{i}}\right] \mathbf{1}\left(\left\|x_{i}\right\|>\mathrm{a}\right) \mathrm{d} x_{i},
$$

which equals

$$
\begin{aligned}
\eta \mathbb{E}^{! o}\left[\mathrm{I}^{n}\right] & =\sum_{\left(p_{1}, \ldots, p_{k}\right) \in \mathcal{P}(n)}(2 \pi)^{k} \prod_{i=1}^{k} \mathbb{E}\left[\mathrm{h}^{p_{i}}\right] \frac{\mathrm{a}^{-p_{i} \alpha+2}}{p_{i} \alpha-2} \\
& =\sum_{\left\{p_{1}, \ldots, p_{k}\right\} \in \mathcal{P}(n)}(2 \pi)^{k} \mathrm{a}^{-n \alpha+2 k} \prod_{i=1}^{k} \frac{\mathbb{E}\left[\mathrm{h}^{p_{i}}\right]}{p_{i} \alpha-2} \\
& \stackrel{(a)}{\leq} \sum_{\left(p_{1}, \ldots, p_{k}\right) \in \mathcal{P}(n)}\left(\frac{2 \pi}{\alpha-2}\right)^{k} \mathrm{a}^{-n \alpha+2 k} b^{n} n !
\end{aligned}
$$


where $(a)$ follows from the inequality $p_{i} \alpha-2>p_{i}(\alpha-2), p_{i} \geq 1$, Assumption A.5 and the inequality $(n+$ $m) !>n ! m !$. It is a known result that the cardinality of the partitions $|\mathcal{P}(n)| \sim \exp (\pi \sqrt{2 n / 3}) /(4 n \sqrt{3})=$ $g_{n}$ for large $n$ [43]. Hence it follows that

$$
\eta \mathbb{E}^{! o}\left[\mathrm{I}^{n}\right]=O\left(g_{n} \max \left\{\left(\frac{2 \pi}{\alpha-2}\right)^{n}, 1\right\} \mathrm{a}^{-n \alpha+2 n} b^{n} n !\right),
$$

as $n \rightarrow \infty$. Let $1 / \epsilon \in(0, \alpha-2)$ and for notational convenience we assume the worst case, $\frac{2 \pi}{\alpha-2}>1$. Choose $\mathrm{a}=e\left(\frac{2 \pi b}{\alpha-2}\right)^{\epsilon}$. Then

$$
\eta \mathbb{E}^{! o}\left[\mathrm{I}^{n}\right]=O(\underbrace{e^{\pi \sqrt{2 n / 3}-n \alpha+2}\left(\frac{2 \pi b}{\alpha-2}\right)^{-n(\epsilon(\alpha-2)-1)} n !}_{D_{n}}),
$$

as $n \rightarrow \infty$. Hence from Assumption A.1 it follows that

$$
\sum\left|D_{n} c_{n}\right| / n !<\infty
$$

Hence using dominated convergence theorem,

$$
\lim _{\mathrm{a} \rightarrow \infty} \sum_{k=1}^{\infty} \frac{c_{k}}{k !} \frac{\mathbb{E}^{! o}\left[\mathrm{I}^{k+\nu}\right]}{\mathrm{a}^{\alpha \nu}}=\sum_{k=1}^{\infty} \frac{c_{k}}{k !} \lim _{\mathrm{a} \rightarrow \infty} \frac{\mathbb{E}^{! o}\left[\mathrm{I}^{k+\nu}\right]}{\mathrm{a}^{\alpha \nu}} .
$$

From Lemma 2, it can be observed that

$$
\rho_{\mathrm{a}}^{(k+1)}\left(\mathrm{a} x_{1}, \ldots, \mathrm{a} x_{k}\right) \sim \mathrm{a}^{-2(k+1)} \tilde{\rho}^{(k+1)}\left(x_{1}, \ldots, x_{k}\right) .
$$

Using the substitution $x_{i} \rightarrow \mathrm{a} x_{i}$ in (33), we obtain

$$
\begin{aligned}
& \eta \mathbb{E}^{! o}\left[\mathrm{I}^{n}\right] \sim \sum_{\left(p_{1}, \ldots, p_{k}\right) \in \mathcal{P}(n)} \mathrm{a}^{-2(k+1)} \int \tilde{\rho}^{(k+1)}\left(x_{1}, \ldots, x_{k}\right) \\
& \cdot \prod_{i=1}^{k} \mathrm{a}^{-\alpha p_{i}+2}\left\|x_{i}\right\|^{-\alpha p_{i}} \mathbb{E}\left[\mathrm{h}^{p_{i}}\right] \mathrm{d} x_{i} .
\end{aligned}
$$

Hence we obtain,

$$
\begin{aligned}
\lim _{\mathrm{a} \rightarrow 0} \frac{\mathbb{E}^{! o}\left[\mathrm{I}^{n}\right]}{\mathrm{a}^{\alpha \nu}} \sim \lim _{\mathrm{a} \rightarrow 0} \pi \mathrm{a}^{\alpha(n-\nu)} \sum_{\left(p_{1}, \ldots, p_{k}\right) \in \mathcal{P}(n)} \\
\qquad \tilde{\rho}^{(k+1)}\left(x_{1}, \ldots, x_{k}\right) \prod_{i=1}^{k}\left\|x_{i}\right\|^{-\alpha p_{i}} \mathbb{E}\left[\mathrm{h}^{p_{i}}\right] \mathrm{d} x_{i},
\end{aligned}
$$

which is equal to zero for $n>\nu$. Hence

$$
\lim _{\mathrm{a} \rightarrow \infty} \frac{\mathbb{E}^{! o}\left[\mathrm{I}^{k+\nu}\right]}{\mathrm{a}^{\alpha \nu}}=0, \quad k>0 .
$$

Using (34) the theorem is proved. 


\section{APPENDIX F}

\section{PROOF OF LEMMA 4}

Proof: We first observe that as $\epsilon \rightarrow 0, f^{*}(\epsilon) \rightarrow 0$. We begin by proving the case when $f(x)>f_{1}(x)$ in the neighborhood of zero. See Figure 3 for the rest of the proof. The case when $f_{1}(x)>f(x)$ around

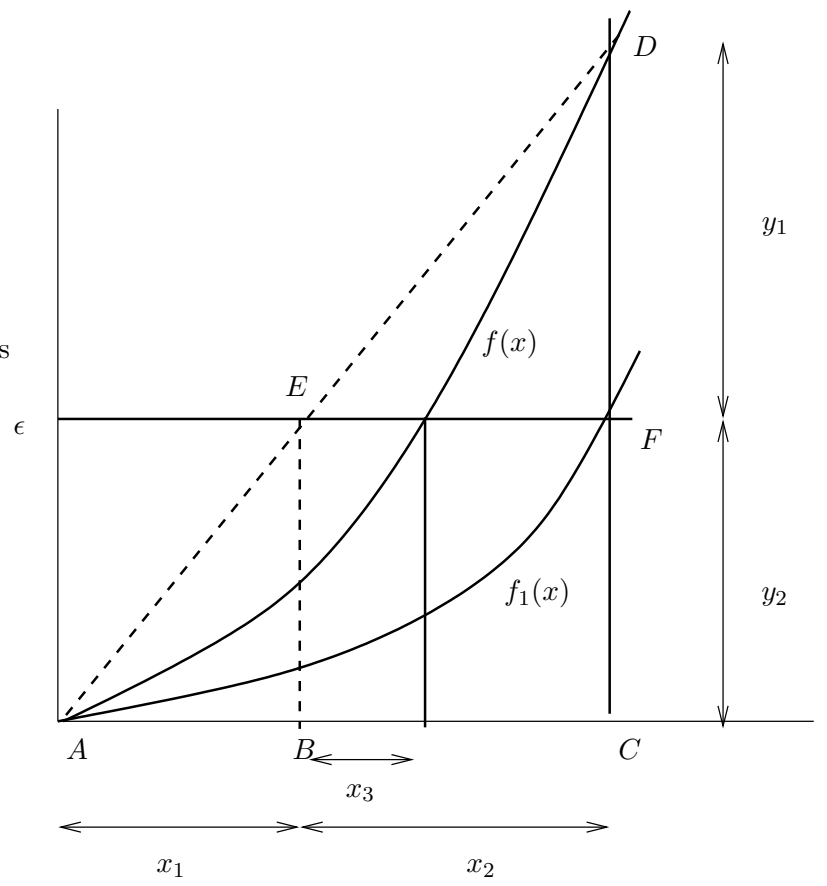

Fig. 3. By the local convexity of the function $f_{1}(x)$, it follows that $f_{1}(x)$ lies below the line $A D$. For small $x, y_{2}=f_{1}(x)$ and $y_{1}=o\left(f_{1}(x)\right)$, i.e., $y_{1} / y_{2} \rightarrow 0$. We require to prove that $\left(x_{2}-x_{3}\right) /\left(x_{1}+x_{3}\right) \rightarrow 0$, which is true if $x_{2} / x_{1} \rightarrow 0$. But by the congruency of triangles $A B E$ and $A C D$ and the congruency of the triangles $D E F$ and $D A C$ it is easy to show that $y_{2} / y_{1}=x_{1} / x_{2}$, hence proving the claim.

$x=0$ can be dealt with in a similar way.

\section{APPENDIX G}

\section{PROOF OF THEOREM 7}

In this section we begin with a few lemmas which are required to prove Theorem 7.

Lemma 6. For any stationary process $\Phi_{t}$, if

$$
\frac{\eta^{-1} \int_{\mathbb{R}^{2}} \rho_{\eta}^{(2)}(x) \Delta^{2}(x) \mathrm{d} x}{\sigma_{\eta}}>2,
$$

then

$$
\frac{\mathcal{G}[\exp (-\Delta(x))]}{1-\mu_{\eta}+\sigma_{\eta} / 2}>1
$$


Proof: We have

$$
\begin{aligned}
\mathcal{G}[\exp (-\Delta(x))] & =\mathbb{E}^{! o}\left[\prod_{\mathrm{x} \in \Phi_{t}} 1-(1-\exp (-\Delta(x)))\right] \\
& \stackrel{(a)}{>} 1-\mathbb{E}^{! o} \sum_{\mathrm{x} \in \Phi_{t}} 1-\exp (-\Delta(\mathrm{x})) \\
& =1-\eta^{-1} \int_{\mathbb{R}^{2}} \rho_{\eta}^{(2)}(x)(1-\exp (-\Delta(x))) \mathrm{d} x
\end{aligned}
$$

where $(a)$ follows from $\prod 1-a_{i} \geq 1-\sum a_{i}$. Using the expansion of $\exp (-x)$, we have $\mathcal{G}[\exp (-\Delta(x))]$ is greater than

$$
1-\mu_{\eta}+\sum_{m=2}^{\infty} \frac{(-1)^{m} \eta^{-1}}{m !} \int_{\mathbb{R}^{2}} \rho_{\eta}^{(2)}(x) \Delta^{m}(x) \mathrm{d} x .
$$

So it is sufficient to prove that the summation is greater than $\kappa$. By the inequality

$$
(x-1)+\exp (-x) \geq \frac{x^{2}}{4}, \quad x \in[0,1]
$$

it is sufficient to show

$$
(4 \eta)^{-1} \int_{\mathbb{R}^{2}} \rho_{\eta}^{(2)}(z) \Delta^{2}(z) \mathrm{d} z \geq \sigma_{\eta} / 2 .
$$

which follows from the assumption.

We now show that for any positive density of transmitters $\eta>0$, the success probability is strictly less than one.

\section{Lemma 7.}

$$
\bar{\eta}=\sup \{\eta \geq 0: 1-\mathcal{G}[\exp (-\Delta(x))]<\epsilon\}
$$

tends to zero as $\epsilon \rightarrow 0$.

Proof: From the definition of the reduced probability generating functional,

$$
\begin{aligned}
\mathcal{G}[\exp (-\Delta(x))] & =\mathbb{E}^{! o} \exp \left(-\sum_{\mathbf{x} \in \Phi_{\eta}} \Delta(x)\right) \\
& \stackrel{(a)}{\geq} \exp \left(-\mu_{\eta}\right)
\end{aligned}
$$

where $(a)$ follows from Jensen's inequality. So it is sufficient to prove that $\sup \eta$ with the constraint $\exp \left(-\mu_{\eta}\right)>1-\epsilon$ tends to zero as $\epsilon \rightarrow 0$. But since $\mu_{\eta}$ is the average of $\sum_{\mathbf{x} \in \Phi_{t}} \Delta(x)$ with respect to the Palm distribution and since $\Delta(x)>0$, a necessary condition for $\mu_{\eta} \rightarrow 0$ is for the density $\eta$ to tend to zero.

We now return to the proof of Theorem 7. From (29), it suffices to prove $\mathrm{TC}_{\mathrm{u}}(\epsilon)=\mathrm{TC}_{1}(\epsilon)+o\left(\mathrm{TC}_{1}(\epsilon)\right)$. The upper bound $\mathrm{TC}_{\mathrm{u}}(\epsilon)$ is equal to the supremum value of $\eta$ so that

$$
\min \left\{1-\mu_{\eta}+\sigma_{\eta} / 2, \mathcal{G}(\exp (-\Delta(x)))\right\}>1-\epsilon .
$$




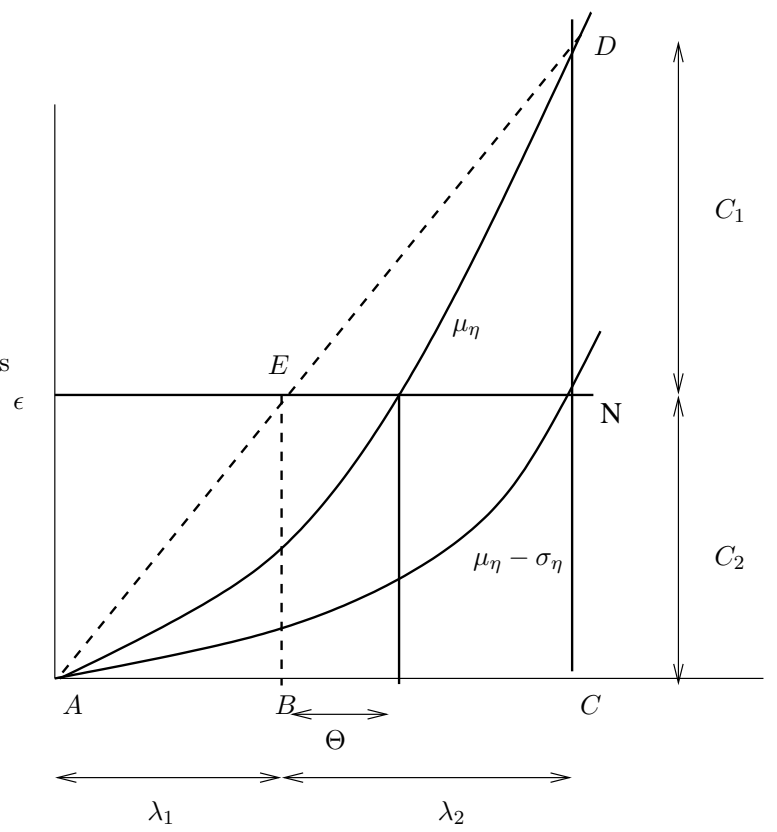

Fig. 4. Proof for Theorem 7. Observe that the triangle $A B E$ is congruent to the triangle $A C D$.

This condition implies $\eta \rightarrow 0$ as $\epsilon \rightarrow 0$ by Lemma 7. Since $\eta$ should be small, it follows from Lemma 6 that

$$
\min \left\{1-\mu_{\eta}+\sigma_{\eta} / 2, \mathcal{G}(\exp (-\Delta(x)))\right\}=1-\mu_{\eta}+\sigma_{\eta} / 2
$$

So the upper bound translates to finding the maximum $\eta$ such that $\mu_{\eta}-\sigma_{\eta} / 2<\epsilon$. Also by our Assumption A.2, $\mu_{\eta}$ is locally convex in the neighborhood of $\eta=0$. See Figure 4 where the upper and lower bounds are illustrated. It suffices to prove $\lim _{\eta \rightarrow 0}\left(\lambda_{2}-\Theta\right) /\left(\lambda_{1}+\Theta\right)=0$. Also Assumption A.3, implies $\lim _{\eta \rightarrow 0} C_{1} /\left(C_{1}+C_{2}\right)=0$. Hence by the congruency of the triangles $A B E$ and $A C D$, it follows that $\lambda_{2} / \lambda_{1}$ tends to zero, which proves the theorem.

\section{REFERENCES}

[1] M. Haenggi, J. G. Andrews, F. Baccelli, O. Dousse, and M. Franceschetti, "Stochastic geometry and random graphs for the analysis and design of wireless networks," IEEE Journal on Sel. Areas in Communications, vol. 27, pp. 1029 -1046, Sept. 2009.

[2] F. Baccelli and B. Blaszczyszyn, "Stochastic geometry and wireless networks. Volume II-applications," NOW: Foundations and Trends in Networking, vol. 4, no. 1-2, pp. 1-312, 2009.

[3] E. S. Sousa and J. A. Silvester, "Optimum transmission ranges in a direct-sequence spread spectrum multihop packet radio network," IEEE Journal on Sel. Areas in Communications, pp. 762-771, June 1990.

[4] J. Ilow and D. Hatzinakos, "Analytic alpha-stable noise modeling in a Poisson field of interferers or scatterers," IEEE Trans. on Signal Processing, vol. 46, pp. 1601-1611, June 1998.

[5] F. Baccelli and S. Zuyev, "Stochastic geometry models of mobile communication networks," in Frontiers in queueing, pp. 227-243, CRC Press, 1997. 
[6] F. Baccelli, M. Klein, M. Lebourges, and S. Zuyev, "Stochastic geometry and architecture of communication networks," J. Telecommunication Systems, vol. 7, no. 1, pp. 209-227, 1997.

[7] M. Zorzi and S. Pupolin, "Optimum transmission ranges in multihop packet radio networks in the presence of fading," IEEE Trans. on Communications, vol. 43, pp. 2201-2205, July 1995.

[8] M. Hellebrandt and R. Mathar, "Cumulated interference power and bit-error-rates in mobile packet radio," Wireless Networks, Springer, vol. 3, no. 3, pp. 169-172, 1997.

[9] M. Haenggi and R. K. Ganti, "Interference in large wireless networks," NOW: Foundations and Trends in Networking, vol. 3, no. 2, pp. 127-248, 2009.

[10] J. Venkataraman, M. Haenggi, and O. Collins, "Shot noise models for the dual problems of cooperative coverage and outage in random networks," in Proc., Allerton Conf. on Comm., Control, and Computing, (Monticello, IL), Sept. 2006.

[11] C. C. Chan and S. V. Hanly, "Calculating the outage probability in a CDMA network with spatial Poisson traffic," IEEE Trans. on Veh. Technology, vol. 50, pp. 183-204, Jan. 2001.

[12] M. Z. Win, P. C. Pinto, and L. A. Shepp, “A mathematical theory of network interference and its applications," Proceedings of the IEEE, vol. 97, pp. 205-230, Feb. 2009.

[13] F. Baccelli, P. Miihlethaler, and B. Blaszczyszyn, "Stochastic analysis of spatial and opportunistic ALOHA," IEEE Journal on Sel. Areas in Communications, vol. 27, pp. 1105-1119, Sept. 2009.

[14] F. Baccelli, B. Blaszczyszyn, and P. Muhlethaler, "An ALOHA protocol for multihop mobile wireless networks," IEEE Trans. on Info. Theory, vol. 52, Feb. 2006.

[15] S. Weber, J. G. Andrews, and N. Jindal, "The effect of fading, channel inversion, and threshold scheduling on ad hoc networks," IEEE Trans. on Info. Theory, vol. 53, pp. 4127-49, Nov. 2007.

[16] S. Weber, X. Yang, J. G. Andrews, and G. de Veciana, "Transmission capacity of wireless ad hoc networks with outage constraints," IEEE Trans. on Info. Theory, vol. 51, pp. 4091-4102, Dec. 2005.

[17] S. Weber, J. G. Andrews, and N. Jindal, "An overview of the transmission capacity of wireless networks," IEEE. Trans. on Communications, submitted, available at: arxiv.org/abs/0809.0016.

[18] M. Kountouris and J. G. Andrews, "Transmission capacity scaling of SDMA in wireless ad hoc networks," in IEEE Information Theory Workshop, (Taormina, Italy), Oct. 2009.

[19] S. Weber, J. G. Andrews, X. Yang, and G. de Veciana, "Transmission capacity of wireless ad hoc networks with successive interference cancellation,” IEEE Trans. on Info. Theory, vol. 53, pp. 2799-2814, Aug. 2007.

[20] K. Huang, J. G. Andrews, R. W. Heath, D. Guo, and R. Berry, "Spatial interference cancellation for mobile ad hoc networks: Perfect CSI," in Proc., IEEE Globecom, pp. 1-5, Dec. 2008.

[21] R. Vaze and R. Heath, "Transmission capacity of wireless ad-hoc networks with multiple antennas using multi-mode precoding and interference cancelation," in Proc., IEEE Signal Proc. Adv. in Wireless Comm. (SPAWC), (Perugia, Italy), pp. $404-408$, June 2009.

[22] R. K. Ganti and M. Haenggi, "Interference and outage in clustered wireless ad hoc networks," IEEE Trans. on Info. Theory, vol. 55, pp. 4067-4086, Sept. 2009.

[23] M. Durvy, O. Dousse, and P. Thiran, "Self-organization properties of CSMA/CA systems and their consequences on fairness,” IEEE Trans. on Info. Theory, vol. 55, pp. 931-943, Mar. 2009.

[24] J. Silvester and L. Kleinrock, "On the capacity of multihop slotted ALOHA networks with regular structure," IEEE Trans. on Communications, vol. 31, pp. 974-982, Aug. 1983.

[25] G. Ferrari and O. K. Tonguz, "MAC protocols and transport capacity in ad hoc wireless networks: Aloha versus PR-CSMA," in Proc., IEEE MILCOM, vol. 2, pp. 1311-1318, Oct. 2003.

[26] M. Haenggi, “Outage, local throughput, and capacity of random wireless networks," IEEE Trans. on Wireless Communications, vol. 8, pp. 4350-4359, Aug. 2009. 
[27] R. Mathar and J. Mattfeldt, "On the distribution of cumulated interference power in Rayleigh fading channels," Wireless Networks, Springer, vol. 1, no. 1, pp. 31-36, 1995.

[28] A. Hasan and J. G. Andrews, "The guard zone in wireless ad hoc networks," IEEE Trans. on Wireless Communications, vol. 6, pp. 897-906, Mar. 2007.

[29] M. Kaynia and N. Jindal, "Performance of ALOHA and CSMA in spatially distributed wireless networks," in Proc., IEEE Intl. Conf. on Communications, pp. 1108-1112, May 2008.

[30] M. Kaynia, G. E. Oien, and N. Jindal, "Joint transmitter and receiver carrier sensing capability of CSMA in MANETs," in Proc., Intl. Conf. on Wireless Communications and Signal Processing, pp. 1-5, Nov. 2009.

[31] R. Giacomelli, R. K. Ganti, and M. Haenggi, "Outage probability of general ad hoc networks in the high-reliability regime," Submitted to IEEE/ACM Transactions on Networking, Feb. 2010. Available at http://arxiv.org/abs/1003.0248.

[32] R. Giacomelli, R. K. Ganti, and M. Haenggi, "Asymptotic outage analysis of general motion-invariant ad hoc networks," in Proc., Information Theory and its Applications, (San Diego, USA), Jan. 2010.

[33] R. K. Ganti and J. G. Andrews, "A new method for computing the transmission capacity of non-Poisson wireless networks," in Proc., IEEE Intl. Symposium on Information Theory, (Austin, USA), June 2010.

[34] D. Stoyan, W. S. Kendall, and J. Mecke, Stochastic Geometry and its Applications. Wiley series in probability and mathematical statistics, New York: Wiley, second ed., 1995.

[35] D. J. Daley and D. Vere-Jones, An Introduction to the Theory of Point Processes. New York: Springer, second ed., 2002.

[36] D. R. Cox and V. Isham, Point Processes. London and New York: Chapman and Hall, 1980.

[37] D. L. Snyder and M. L. Miller, Random Point Processes in Time and Space. Springer, 1991.

[38] X. Yang and G. de Veciana, "Inducing multiscale spatial clustering using multistage MAC contention in spread spectrum ad hoc networks," IEEE/ACM Trans. on Networking, vol. 15, no. 6, pp. 1387-1400, 2007.

[39] J. G. Andrews, S. Weber, and M. Haenggi, “Ad hoc networks: To spread or not to spread?, IEEE Communications Magazine, vol. 45, pp. 84-91, Dec. 2007.

[40] A. Hunter, J. G. Andrews, and S. Weber, "Transmission capacity of ad hoc networks with spatial diversity," IEEE Trans. on Wireless Communications, vol. 7, pp. 5058-5071, Dec. 2008.

[41] B. Bollobás and O. Riordan, Percolation. Cambridge Univ. Pr., 2006.

[42] G. B. Folland, Real Analysis, Modern Techniques and Their Applications. Wiley, 2 ed., 1999.

[43] H. Rademacher, "On the partition function p(n)," Proceedings of the London Mathematical Society, vol. 2, no. 1, p. 241, 1938. 